\title{
An RGDKGE-Containing Cryptic Collagen Fragment Regulates Phosphorylation of Large Tumor Suppressor Kinase-1 and Controls Ovarian Tumor Growth by a Yes-Associated Protein-Dependent Mechanism
}

\author{
XiangHua Han, Jennifer M. Caron, Christine W. Lary, Pradeep Sathyanarayana, Calvin Vary, and Peter C. Brooks
}

From the Maine Medical Center Research Institute, Center for Molecular Medicine, Scarborough, Maine

\author{
Accepted for publication \\ November 17, 2020. \\ Address correspondence to \\ Peter C. Brooks, Ph.D., Maine \\ Medical Center Research \\ Institute, Center for Molecu- \\ lar Medicine, 81 Research \\ Dr., Scarborough, ME \\ 04074. E-mail: brookp1@ \\ mmc.org.
}

\begin{abstract}
The growth and spread of malignant tumors, such as ovarian carcinomas, are governed in part by complex interconnected signaling cascades occurring between stromal and tumor cells. These reciprocal cross-talk signaling networks operating within the local tissue microenvironment may enhance malignant tumor progression. Understanding how novel bioactive molecules generated within the tumor microenvironment regulate signaling pathways in distinct cellular compartments is critical for the development of more effective treatment paradigms. Herein, we provide evidence that blocking cellular interactions with an RGDKGE-containing collagen peptide that selectively binds integrin $\beta 3$ on ovarian tumor cells enhances the phosphorylation of the hippo effector kinase large tumor suppressor kinase-1 and reduces nuclear accumulation of yes-associated protein and its target gene c-Myc. Selectively targeting this RGDKGE-containing collagen fragment inhibited ovarian tumor growth and the development of ascites fluid in vivo. These findings suggest that this bioactive collagen fragment may represent a previously unknown regulator of the hippo effector kinase large tumor suppressor kinase-1 and regulate ovarian tumor growth by a yes-associated protein-dependent mechanism. Taken together, these data not only provide new mechanistic insight into how a unique collagen fragment may regulate ovarian cancer, but in addition may help provide a useful new alternative strategy to control ovarian tumor progression based on selectively disrupting a previously unappreciated signaling cascade. (Am J Pathol 2021, 191: 527-544; https://doi.org/10.1016/j.ajpath.2020.11.009)
\end{abstract}

The stromal microenvironment, which is composed of a variety of diverse cell types, secreted factors, and a complex interconnected network of extracellular matrix (ECM) molecules, can exert profound effects on tumors. ${ }^{1-3}$ Cellular interactions with specific ECM components, such as collagen, play central roles in controlling many protumorigenic signaling programs that govern cellular proliferation, survival, migration, and invasion. Dysregulation of these processes underlies many of the altered phenotypic characteristics observed in malignant tumors. ${ }^{4-6}$ Our laboratory has been focused on developing a more detailed cellular and molecular understanding of how both stromal and tumor cells utilize signaling cascades that are uniquely stimulated by cellular interactions with cryptic or hidden regions within ECM proteins.
Our prior studies have identified several matriximmobilized cryptic integrin binding sites within ECM molecules that actively regulate angiogenesis, tumor growth, and metastasis. ${ }^{7-9}$ A variety of studies suggest that, in addition to structurally altered ECM proteins that remain immobilized within the insoluble matrix, bioactive fragments of ECM proteins can also be released in a soluble form. ${ }^{10-12}$ Elevated levels of some of these peptides have

Supported in part by NIH grant CA196739 (P.C.B.); the Office of the Assistant Secretary of Defense for Health Affairs, through the Ovarian Cancer Research Program award W81XWH-17-1-0099 (P.C.B.); the Northern New England Clinical and Translational Research Center grant U54GM115516; the Mesenchymal and Neural Regulation of Metabolic Networks grant P20GM121301; and the Maine Medical Center.

Disclosures: P.C.B. and C.V. hold equity positions in CryptoMedix, Inc. 
been shown to correlate with a more aggressive tumor phenotype in human subjects. ${ }^{12}$ In fact, subsequent studies have now shown that elevated levels of a defined subset of soluble collagen fragments detected in a cohort of cancer patients correlate with resistance to anti-cytotoxic T-lymphocyte associated protein-4 immune checkpoint therapy. ${ }^{13}$ These and several other studies are consistent with the possibility that a subset of soluble ECM fragments may not only represent clinically useful biomarkers for studying disease progression and resistance to therapy, but may also play fundamental roles in controlling angiogenesis, inflammation, and tumor growth. In this regard, we previously identified an endogenously expressed soluble collagen fragment generated by a subset of M2-like macrophages. ${ }^{11}$ This soluble RGDKGE-containing collagen fragment stimulated angiogenesis and inflammation in vivo. ${ }^{11}$ These observations were surprising given that many studies have shown that argine-glycine-aspartic acid-containing peptides often exhibit the opposite effect, and inhibit angiogenesis and tumor growth. ${ }^{14,15}$ This unexpected pro-angiogenic activity appeared to depend in part on the unique flanking sequences surrounding the core RGD motif, as other RGD-containing collagen peptides lacking this motif failed to significantly promote angiogenesis or inflammation in this model. ${ }^{11}$

Although this RGDKGE-containing collagen fragment selectively bound to vascular endothelial cells and stimulated angiogenesis dependent in part on the transcriptional coactivator yes-associated protein (YAP), it is not known whether this collagen fragment is generated in malignant tumors or whether it plays a functional role in tumor growth in vivo. Although some studies have implicated YAP in promoting the growth of different tumor types, including ovarian carcinomas, ${ }^{16,17}$ other studies have suggested that YAP signaling might exhibit tumor suppressive activity, depending on the specific cell type and tissue microenvironment. ${ }^{18,19}$ In fact, recent studies suggest that YAP activity in peritumoral hepatocytes may suppress the growth of liver tumors. ${ }^{19}$ Thus, a more indepth molecular understanding of how YAP activity is controlled in context-dependent pathologic processes may allow for the development of innovative new approaches to selectively regulate YAP within distinct tissue microenvironments. A wide array of molecular regulators and mechanisms have been identified that govern the activation and nuclear accumulation of YAP. Among the mechanisms by which nuclear YAP accumulation is thought to be controlled include the canonical hippo signaling cascade. $^{20,21}$ Although the ability of the core kinases within the hippo signaling cascade to control YAP activity is well documented, little is known concerning the possibility that soluble collagen-derived fragments may regulate the hippo pathway or whether targeting these extracellular regulators may be exploited to selectively control YAP activity during tumor growth in vivo. Herein, we provide evidence that blocking a soluble RGDKGE-containing collagen fragment can regulate the large tumor suppressor kinase-1 (LATS1), a core effector molecule within the hippo pathway. We show that ovarian cancer cell interactions with an RGDKGE collagen peptide activate $\beta 3$ integrin and reduce the level of inhibitory phosphorylation of YAP and enhance its nuclear accumulation. Moreover, selectively blocking cellular interactions with the RGDKGE collagen peptide enhanced the phosphorylation of LATS1 and YAP, reduced nuclear accumulation of YAP, and reduced the levels of the YAP target gene c-Myc. More important, selectively blocking cellular interactions with the RGDKGE-containing collagen fragment inhibited the development of ascites fluid and ovarian tumors growing in vivo. These findings indicate that a soluble RGDKGE-containing collagen fragment may represent a previously unknown regulator of the hippo effector kinase LATS1 that plays a role in controlling ovarian tumor growth. These data not only provide new mechanistic insight into how an endogenously generated bioactive collagen fragment may regulate ovarian cancer, but also provide an alternative strategy for the development of new therapeutic approaches to control ovarian tumor progression.

\section{Materials and Methods}

\section{Cells and Cell Culture}

Murine ID8-vascular endothelial growth factor (VEGF) ovarian carcinoma cells ${ }^{22}$ were obtained from Dr. Philip Greenberg (University of Washington School of Medicine, Seattle, WA). ID8-VEGF cells were cultured in Dulbecco's modified Eagle's medium in the presence of $4 \%$ fetal bovine serum, $1.0 \%$ penicillin-streptomycin, $1.0 \%$ sodium pyruvate, and $1 \%$ of insulin-transferrin-sodium selenite media supplements. SKOV-3 ovarian carcinoma cells were obtained from ATCC (Manassas, VA) and cultured in RPMI 1640 medium in the presence of 5\% fetal bovine serum, $1 \%$ penicillin-streptomycin, and $1 \%$ sodium pyruvate. RAW 264.7 cells were obtained from ATCC and cultured in Dulbecco's modified Eagle's medium in the presence of $10 \%$ fetal bovine serum, $1 \%$ penicillin-streptomycin, and $1 \%$ sodium pyruvate.

\section{Cell Culture in 3D Type I Collagen Gels}

Briefly, ID8-VEGF cells were mixed with $1 \mathrm{~mL}$ of threedimensional (3D) collagen gel-forming solution (Pure Col EZ 3D Type I Collagen Gel Solution; Advanced Biomatrix, San Diego, CA) and incubated for 1 hour at $37^{\circ} \mathrm{C}$ until gel formation. After adding $1 \mathrm{~mL}$ of serum-free Dulbecco's modified Eagle's medium on the top of 3D collagen gel, cells were incubated for 72 hours and the conditioned medium was collected for Western blot analysis. 


\section{Animals}

C57BL/6J female mice (6 to 8 weeks old) were obtained from the Jackson Laboratory (Bar Harbor, ME). NCRNU-F female mice (6 to 8 weeks old) were obtained from Taconic Biosciences (Germantown, NY). Mice were housed in Maine Medical Center Research Institute (Scarborough, ME) pathogen-free air barrier facility, and all animal handling and procedures were approved by the Maine Medical Center Institutional Animal Care and Use Committee.

\section{Reagents, Chemicals, and Antibodies}

Bovine serum albumin, insulin-transferrin-sodium selenite media supplements, and crystal violet were obtained from Sigma (St. Louis, MO). Anti-YAP1, anti-phosphorylated YAP (Ser127), anti-LATS1, anti-phosphorylated LATS (Thr1079), anti-rous sarcoma tyrosine protein kinase, anti-phosphorylated SRC (Y416), anti-phosphorylated mammalian STE20-like protein kinase-1 (MST1)-Thr-183, anti-integrin $\beta 3$, and anti-TATA-binding protein antibodies were all obtained from Cell Signaling (Danvers, MA). Anti-phosphorylated integrin $\beta 3$ (Ser747) and anti- $\beta$-actin were obtained from Santa Cruz Biotechnology (Dallas, TX). Anti-MST1 antibody was obtained from Novus Biologicals (Centenial, CO). Anti-CD-31 antibody was obtained from Abcam (Cambridge, MA). Monoclonal antibody (Mab) XL313 and non-specific control antibody were obtained from Bio X Cell (Lebanon, NH). P2 peptide (CQGPRGDKGEC) and control peptide (CP; CQGPGGAAGGC) were from QED Bioscience (San Diego, CA). Alexa 594-labeled secondary antibody was from Invitrogen (Carlsbad, CA). The SRC inhibitor dasatinib was from Selleckchem (San Diego, CA). Pure Col EZ 3D Type I Collagen Gel Solution was obtained from Advanced Biomatrix. Cell proliferation assay kit was obtained from EMD Millipore (Temecula, CA). Poly-L-lysine was obtained from ScienCell Research Laboratories (Carlsbad, CA). Formaldehyde was obtained from Polysciences Inc. (Warrington, PA).

\section{Western Blot Analysis}

ID8-VEGF or SKOV-3 cells from subconfluent culture were serum starved in suspension for 1 hour at $37^{\circ} \mathrm{C}$, and seeded on CP or RGDKGE-containing peptide (P2) coated plates in the presence or absence of Mab XL313 or control antibody $(100 \mu \mathrm{g} / \mathrm{mL})$ for 5 or 15 minutes under the defined experimental conditions and then lysed in radioimmunoprecipitation assay lysis buffer with $1 \times$ protease inhibitor cocktail, $2 \mathrm{mmol} / \mathrm{L}$ of phenylmethylsulfonyl fluoride, and $1 \mathrm{mmol} / \mathrm{L}$ of sodium orthovanadate (Santa Cruz Biotechnology, Dallas, TX). Equal amounts of cell lysate were separated by SDS-PAGE. Membranes were probed with the indicated antibodies. Western blot analyses were performed at least three to four times and visualized by chemiluminescence detection. Quantification of the mean relative changes in proteins was performed using downloaded ImageJ software version 5.0 (NIH, Bethesda, MD; http://imagej.nih.gov/ij). ${ }^{9}$

\section{Nuclear Protein Extraction}

Subconfluent ID8-VEGF cells were washed and serum starved for 1 hour at $37^{\circ} \mathrm{C}$, then seeded on 6-well plates coated with CP $(100 \mu \mathrm{g} / \mathrm{mL})$, RGDKGE-containing peptide $(\mathrm{P} 2 ; 100 \mu \mathrm{g} / \mathrm{mL})$, or poly-L-lysine $(100 \mu \mathrm{g} / \mathrm{mL})$. In other experiments, subconfluent ID8-VEGF cells were stimulated with CP $(50 \mathrm{ng} / \mathrm{mL})$ or RGDKGE-containing peptide P2 (50 $\mathrm{ng} / \mathrm{mL}$ ) in suspension for 1 hour at $37^{\circ} \mathrm{C}$. In a final set of experiments, ID8-VEGF cells were washed and serum starved for 1 hour at $37^{\circ} \mathrm{C}$, and cells were then seeded on 6-well plates coated $(100 \mu \mathrm{g} / \mathrm{mL})$ with $\mathrm{CP}$ or RGDKGEcontaining peptide (P2). To examine the effects of blocking cellular interactions with the RGDKGE-containing collagen peptide P2 on nuclear accumulation of YAP, cells were seeded on P2 peptide $(100 \mu \mathrm{g} / \mathrm{mL})$-coated 6well plates that were preblocked with $100 \mu \mathrm{g} / \mathrm{mL}$ of Mab XL313, anti-integrin $\beta 3$ antibody, or non-specific control antibody for 30 minutes. To examine the effects of Src on YAP, ID8-VEGF cells from subconfluent culture were washed and serum starved for 30 minutes at $37^{\circ} \mathrm{C}$, and pretreated with $\mathrm{Src}$ inhibitor dasatinib $(1 \mu \mathrm{mol} / \mathrm{L})$ or dimethyl sulfoxide for 30 minutes. Next, cells were seeded on P2 peptide-coated 6-well plates in presence or absence or Mab XL313 antibody or control antibody. After incubating for 1 hour, adherent and nonadherent cells were harvested. The nuclear and cytoplasmic proteins were extracted using NE-PER nuclear and cytoplasmic extraction reagents from Thermo Scientific (Waltham, MA), according to the manufacturer's instructions. The nuclear extractions were examined by Western blot analysis with anti-YAP or anti-TATA-binding protein antibody, and the cytoplasmic extraction was examined by Western blot analysis with total and phosphorylated YAP (Ser-127), total and phosphorylated LATS1 (Thr-1079), or total and phosphorylated MST1 (Thr-183) antibodies.

\section{RNA Isolation, cDNA Synthesis, and Quantitative PCR}

Subconfluent ID8-VEGF cells were washed and serum starved for 1 hour at $37^{\circ} \mathrm{C}$, and cells were seeded on 6-well plates coated with $\mathrm{CP}$ or RGDKGE-containing peptide $\mathrm{P} 2$ $(100 \mu \mathrm{g} / \mathrm{mL})$. To examine the effect of blocking cellular interactions with the RGDKGE-containing peptide P2 on c-Myc, cells were washed and serum starved for 30 minutes at $37^{\circ} \mathrm{C}$ and preblocked with $100 \mu \mathrm{g} / \mathrm{mL}$ of control or Mab XL313 antibodies. Next, cells were seeded on P2 peptide $(100 \mu \mathrm{g} / \mathrm{mL})-$ coated 6-well plates. After 1-hour incubation, cells were lysed using RNA isolation lysis buffer, and RNAs were isolated by using RNase plus mini kit (Qiagen, Carol 
Stream, IL) following the manufacturer's protocol. Total RNA $(1.0 \mu \mathrm{g})$ was synthesized with iScript cDNA synthesis kit from Bio-Rad (Hercules, CA). Equivalent amount of cDNA was used for quantitative RT-PCR (RT-qPCR), and the murine RT-qPCR primers used for mouse $c-M y c$ were $5^{\prime}$ CAGAGGAGGAACGAGCTG-3' (forward) and 5' -TTATGCACCAGAGTTTCGAAGCTGTTCGT- $3^{\prime}$ (reverse); and the primers used for the housekeeping gene mouse $b 2 \mathrm{~m}$ were $5^{\prime}$ CTGACCGGCCTGTATGCTAT-3' (forward) and $5^{\prime}$ CCGTTCTTCCAGCATTTGGAT-3' (reverse). RT-qPCR assays were repeated five times.

\section{Quantification of Tumor Angiogenesis and Levels of Nuclear YAP}

Frozen sections from individual tumors $(n=5$ per condition) were fixed in 50\% methanol and 50\% acetone, and blocked with $2.0 \%$ bovine serum albumin for 1 hour at room temperature. For quantification of angiogenesis, frozen sections of tumors from SKOV-3 or ID8-VEGF ovarian tumors were stained with anti-CD31 (1:300) overnight, followed by incubation with Alexa 594-labeled secondary antibody. Tumor vessel counts were performed on five to eight $\times 200$ microscopic fields from each of four to five independent tumors from each condition. For quantification of the effect of anti-RGDKGE collagen fragment antibody (Mab XL313) or non-specific control antibody on nuclear Yap within the ovarian tumors, frozen sections from SKOV-3 or ID8-VEGF ovarian tumors were fixed with $4 \%$ formaldehyde at room temperature for 15 minutes. Tumor sections were washed three times in $1 \times$ phosphate-buffered saline for 5 minutes each and then blocked with $5 \%$ normal goat serum in $3 \%$ bovine serum albumin/phosphate-buffered saline for 1 hour. Tissue sections were next stained with anti-YAP (1:100) overnight, followed by incubation with Alexa 594-labeled secondary antibody. Nuclear and cytosolic Yap-positive cell counts were performed from four to nine $\times 400$ microscopic fields from each of four to five independent tumors from each condition.

\section{Preparation of Tissue Lysates}

SKOV-3 and ID8-VEGF ovarian tumors $(n=4$ per tumor type) were harvested from nude mice or C57BL/6 mice at day 14 or day 7 , respectively. Individual tumors were snap frozen on dry ice and ground in a cold mortar. Ground up tissues from individual tumors were next mixed with radioimmunoprecipitation assay lysis buffer, and whole tissue lysates were generated. In further experiments, 6- to 8-week-old C57BL/6J female mice ( $n=$ 4) were scarified for skin and peritoneum tissue harvest. Individual skin or peritoneum tissue was snap frozen on dry ice and ground in a cold mortar with radioimmunoprecipitation assay lysis buffer, and whole tissue lysates were generated.

\section{Cell-Binding Assays}

Forty-eight-well nontissue culture plates were coated with $100 \mu \mathrm{g} / \mathrm{mL}$ of $\mathrm{CP}$ or RGDKGE-containing peptide (P2). Tumor cells were suspended in binding buffer (RPMI 1640 medium containing $1 \mathrm{mmol} / \mathrm{L} \mathrm{MgCl} 2,0.2 \mathrm{mmol} / \mathrm{L} \mathrm{MnCl}_{2}$, and $0.5 \%$ bovine serum albumin), and $1 \times 10^{5}$ cells were added to the wells in the presence or absence of $100 \mu \mathrm{g} / \mathrm{mL}$ of Mab XL313, anti-integrin $\beta 3$ antibody, and anti-integrin $\beta 1$ or non-specific normal mouse $\operatorname{IgG}$ control antibody and allowed to bind for 30 to 45 minutes at $37^{\circ} \mathrm{C}$. Nonattached cells were removed, and attached cells were stained with crystal violet. ${ }^{7}$ Cell binding was quantified by measuring the OD of eluted dye. ${ }^{7}$ Cell-binding assays were performed at least three times with triplicate wells per condition.

\section{Cell Growth Assays}

Briefly, ID8-VEGF cells from subconfluent culture were serum starved in suspension for 1 hour at $37^{\circ} \mathrm{C}$, and seeded 2000 cells per well in 96-well microplate in the presence or absence of Mab XL313 $(100 \mu \mathrm{g} / \mathrm{mL})$ or control antibody $(100 \mu \mathrm{g} / \mathrm{mL})$. After incubating the cells for 72 hours, $10 \mu \mathrm{L}$ per well tetrazolium salt 1/ECS solution was added to each well and incubated for 1 hour, and the absorbance was measured by using a microplate reader at $450 \mathrm{~nm}$.

\section{Viral Vectors and Transduction}

Lentiviral vectors (pLKO.1 based) encoding shRNAs specific to YAP or a control nontargeting construct developed by the RNAi Consortium were obtained from GE Healthcare Life Science (Lafayette, CO). The effective targeting sequence of YAP gene was 5'-TTCTTTATCTAGCTTGGTGGC- $3^{\prime}$. Lentivirus was packaged in the recombinant viral vector core facility at Maine Medical Center Research Institute. Cells transduced with the shRNA viruses were selected in growth medium containing $2.5 \mu \mathrm{g} / \mathrm{mL}$ puromycin (Invitrogen) to establish cell pools stably expressing shRNA.

\section{Tumor Growth Assays}

Tumor growth assays were performed as previously described. ${ }^{9}$ Briefly, mice were injected subcutaneously with either $10 \times 10^{6}$ ID8-VEGF cells $(n=8$ to 10 C57BL/6 mice per condition) or $3 \times 10^{6} \mathrm{SKOV}-3$ cells, nontarget SKOV-3 cells, or YAP knockdown SKOV-3 cells $(n=8$ to 10 NCRNU-F mice per condition). Tumors were allowed to form palpable tumors for 3 days, then the mice were injected (intraperitoneally) with Mab XL313 (25 to $250 \mu \mathrm{g}$ per mouse) or non-specific control antibody $(250 \mu \mathrm{g}$ per mouse) three times per week for either 42 days for ID8-VEGF tumor model or 35 days for the SKOV-3 tumor model. Tumor size was measured with 
caliper, and the tumor volume was calculated using the following formula: Volume $=$ Length $^{2} \times$ Width $/ 2$. For ascites tumor assays, $10 \times 10^{6}$ ID8-VEGF cells were injected (intraperitoneally) in C57BL/6J female mice $(n=8)$. Four weeks later, mice were intraperitoneally injected with Mab XL313 or control antibody (100 $\mu \mathrm{g}$ per mouse) twice a week. At the end of the 13-week treatment period, ascites fluid was harvested and the volume was quantified. To estimate tumor burden within the diaphragm, mouse diaphragms were harvested and wet tissue weights were determined ( $n=5$ per group).

\section{Statistical Analysis}

Statistical analysis was performed using the Prism/Graph Pad software version 6.0h (Graphpad Software, Inc., San Diego, CA) for Macintosh computers. Data were analyzed for statistical significance using $t$-test. $P<0.05$ was considered significant.

\section{Results}

Detection of an RGDKGE-Containing Collagen Fragment in Ovarian Tumors and Interaction with Ovarian Carcinoma Cells

Our previous studies identified an endogenously generated cryptic collagen fragment containing the amino acid sequence RGDKGE that could be detected as an approximately $16-\mathrm{kDa}$ soluble fragment in serum-free conditioned medium from M2-like macrophages. ${ }^{11}$ Given these studies, we sought to determine whether this collagen fragment might be generated during tumor growth. To identify potentially relevant tumor models with which to study this collagen fragment, whole tissue lysates of SKOV-3 and ID8-VEGF ovarian tumors growing in mice for the generation of the 16-kDa RGDKGE-containing collagen fragment were examined. The 16-kDa RGDKGE-containing collagen fragment was detected within whole tumor lysates from both SKOV-3 and ID8-VEGF tumors growing in vivo (Figure 1A). To further examine the expression of this 16kDa RGDKGE-containing collagen fragment in normal mouse tissues, whole tissue lysates of skin or peritoneum tissues from individual mice without tumors were examined. Although variable levels of the RGDKGE collagen fragment were detected in two of the four skin samples examined, little, if any, RGDKGE was detected in any of the peritoneum tissues (Figure 1B). These data suggest minimal and differential expression of the $16-\mathrm{kDa}$ RGDKGE collagen fragment within distinct normal tissues. Next, it was assessed whether the 16-kDa RGDKGE collagen fragment could be detcted in conditioned medium derived from ID8-VEGF tumor cells growing in 3D collagen gels. Consistent with our previous findings, ${ }^{11}$ the 16-kDa RGDKGE collagen fragment was readily detected within conditioned medium from M2-like macrophages
(RAW 264.7), whereas none was detected in cell-free medium derived from 3D collagen gels under identical incubation conditions (Figure 1C). Interestingly, the 16-kDa RGDKGE collagen fragment was also detected within conditioned medium derived from ID8-VEGF tumor cells grown within 3D collagen gels (Figure 1C). Given these findings and the fact that the 16-kDa RGDKGE collagen fragment was detected in solid tumors growing in vivo, it was next examined whether ovarian tumor cells could directly express the 16-kDa RGDKGE collagen fragment. To this end, whole cell lysates from ovarian tumor cell lines were examined. Consistent with our previous studies, ${ }^{11}$ the $16-\mathrm{kDa}$ RGDKGE collagen fragment was detected in lysates from RAW 264.7 macrophages (Figure 1D). Interestingly, although low but detectable levels of the 16-kDa RGDKGE collagen fragment were detected in lysates from SKOV-3 tumor cells, relatively higher levels were detected in ID8-VEGF cell lysates (Figure 1D). Taken together, these data suggest that ovarian tumors may represent a biologically relevant tumor type to study the possible impact this collagen fragment might have on tumor growth.

Although our previous studies indicated that vascular endothelial cells could directly bind to the RGDKGE collagen fragment, ${ }^{11}$ it is not known whether ovarian tumor cells themselves also have this binding ability. Therefore, to further study potential roles this collagen fragment might have in ovarian cancer, we assessed whether ovarian carcinoma cells have the capacity to directly interact with an in-house synthetic RGDKGEcontaining collagen peptide P2. ${ }^{11}$ SKOV-3 and ID8VEGF tumor cells readily bound to the $\mathrm{P} 2$ collagen peptide, while exhibiting minimal, if any, binding with the non-specific CP (Figure 1, E and F). More important, Mab XL313, specifically directed to the RGDKGE-containing peptide P2, inhibited cellular binding to P2 compared with a non-specific control antibody (Figure 1, G and H). These data indicate that SKOV-3 and ID8-VEGF tumor cells can interact with the RGDKGE collagen fragment and may represent useful tumor models with which to study its possible functions.

\section{The RGDKGE-Containing Collagen Fragment Activates $\beta 3$ Integrin and Stimulates Enhanced Nuclear Accumulation of YAP}

To determine potential receptors capable of mediating cellular interactions with the RGDKGE-containing collagen peptide P2, cell binding assays were performed in the presence of function-blocking anti-integrin antibodies. Function-blocking antibodies directed to $\beta 3$ integrin significantly inhibited binding of both SKOV-3 and ID8VEGF tumor cells to the P2 collagen peptide by $>80 \%$, whereas a function-blocking antibody directed to $\beta 1$ integrins had no significant inhibitory activity (Figure 2, A and B). These data indicate that $\beta 3$ integrin can serve as a cell 
A

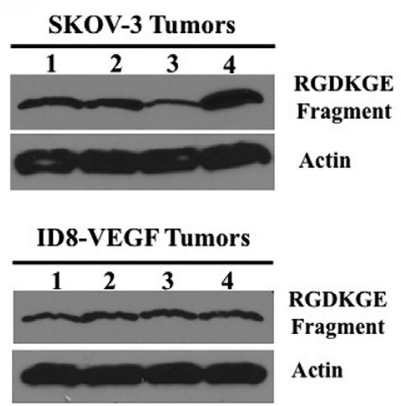

C

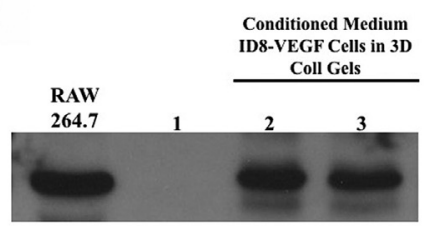

E

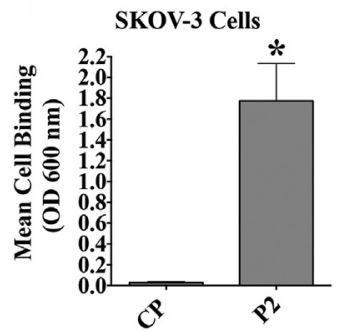

G

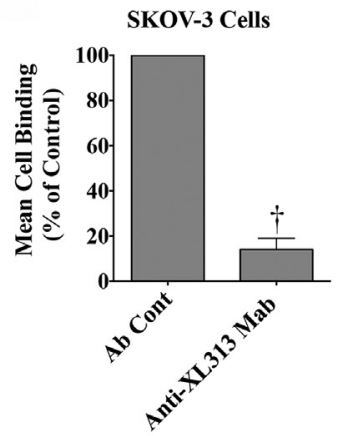

B

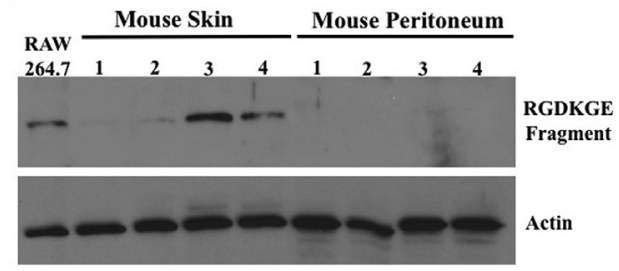

D

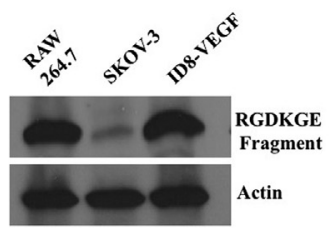

$\mathbf{F}$

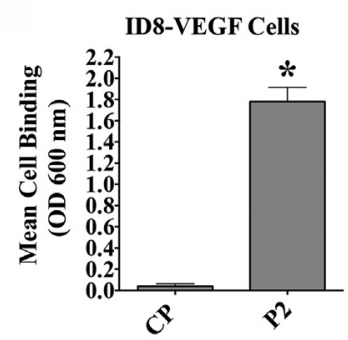

H

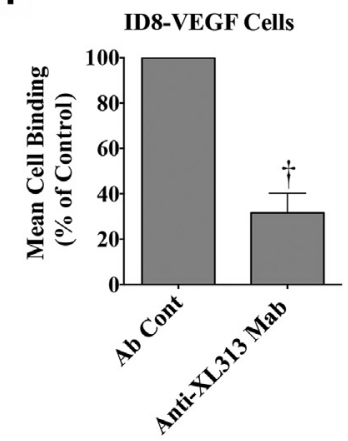

Figure 1 Detection of the RGDKGE-containing collagen fragment in ovarian tumors and its binding to tumor cells. A: Whole tumor lysates were prepared from individual human SKOV-3 (top panels) or murine ID8-vascular endothelial growth factor (VEGF) (bottom panels) tumors grown in mice. Example of Western blot analysis for the levels of the 16-kDa RGDKGE-containing collagen fragment or loading control actin. B: Whole tissue lysates were prepared from normal skin and peritoneum tissues from individual mice. Western blot analysis for the levels of the $16-\mathrm{kDa}$ RGDKGE-containing collagen fragment or loading control actin. C: Western blot analysis of conditioned medium from RAW 264.7 macrophages, control medium derived from three-dimensional (3D) gel in absence of cells (lane 1), or conditioned medium from ID8-VEGF cells cultured in 3D collagen gels (lanes 2 and 3) for the 16-kDa RGDKGE-containing collagen fragment. D: Western blot analysis of whole cell lysates from RAW 264.7 macrophages or SKOV-3 and ID8-VEGF ovarian carcinoma cells for the 16-kDa RGDKGEcontaining collagen fragment or loading control actin. E: Quantification of SKOV-3 cell binding to control peptide (CP) and collagen peptide P2. F: Quantification of ID8-VEGF cell binding to $\mathrm{CP}$ and collagen peptide P2. G: Quantification of SKOV-3 cell binding to collagen peptide $\mathrm{P} 2$ in the presence of non-specific control antibody (Ab Cont) or anti-RGDKGE antibody [Anti-XL313 Monoclonal Antibody (Mab)]. H: Quantification of ID8-VEGF cell binding to collagen peptide P2 in the presence of non-specific Ab Cont or Anti-XL313 Mab. Data are expressed as means \pm SEM (E-H). $n=4$ per group (A and $\mathbf{B}$ ); $n \geq 3$ independent experiments $(\mathbf{E}-\mathbf{H}) .{ }^{*} P<0.05$ versus $\mathrm{CP} ;{ }^{\dagger} P<0.05$ versus $\mathrm{Ab}$ Cont. surface receptor for the $\mathrm{P} 2$ collagen peptide in these ovarian tumor cells.

Next, it was examined whether cellular interactions with the P2 collagen peptide were sufficient to initiate activation of $\beta 3$ integrin, as indicated by phosphorylation of $\beta 3$ at tyrosine 747, which is known to facilitate downstream signaling events. ${ }^{23}$ To study this possibility, ID8-VEGF cells were studied, given their relatively high levels of $\beta 3$ integrin (Figure 2C). To this end, ID8-VEGF cells were stimulated by allowing tumor cells to bind to the P2 collagen peptide or a non-specific $\mathrm{CP}$, and cell lysates were prepared. Tumor cell binding to the P2 collagen peptide significantly enhanced tyrosine phosphorylation of $\beta 3$ integrin by approximately 50\% (Figure 2, D and E). In contrast, blocking binding of $\mathrm{P} 2$ to the ovarian tumor cells with Mab XL313 inhibited $\beta 3$ integrin phosphorylation (Figure 2, F and G). These data indicate that the P2 collagen peptide can directly regulate $\beta 3$-integrin activation, whereas Mab XL313 selectively inhibits this $\beta 3$-integrin activation.

Interestingly, our previous studies suggested that the RGDKGE collagen peptide could stimulate enhanced nuclear accumulation of YAP in endothelial cells. ${ }^{11}$ Given the ability of the RGDKGE collagen fragment to bind and activate $\beta 3$-integrin in ovarian carcinoma cells, it was determined whether $\beta 3$-integrin receptor may also play a role in controlling P2 peptide-stimulated nuclear YAP accumulation in tumor cells. ID8-VEGF cells were stimulated with P2 collagen peptide and examined the effects that 


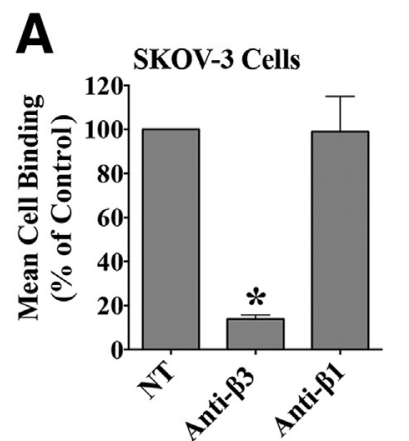

B
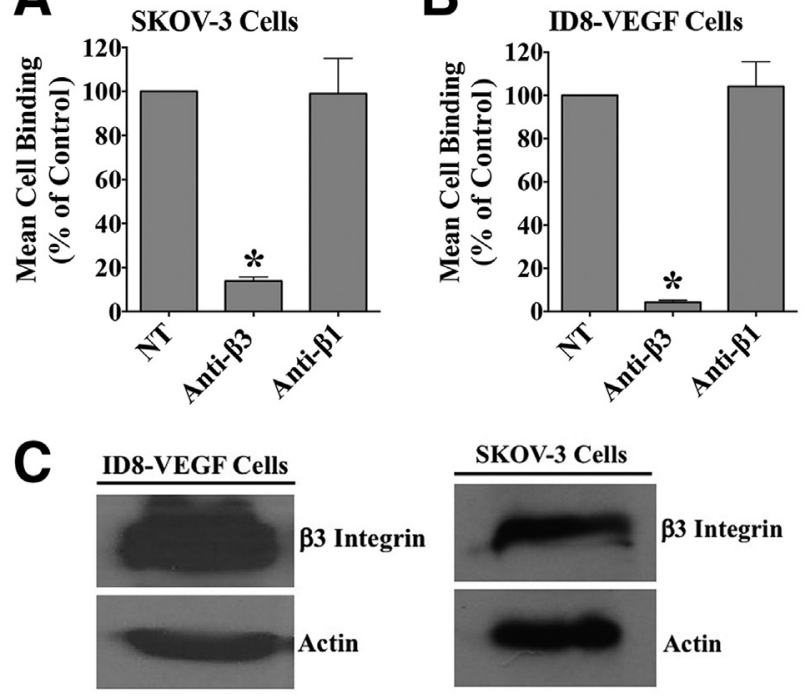

D

ID8-VEGF Cells

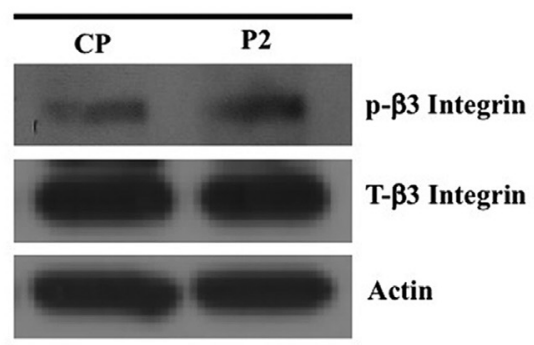

F

ID8-VEGF Cells

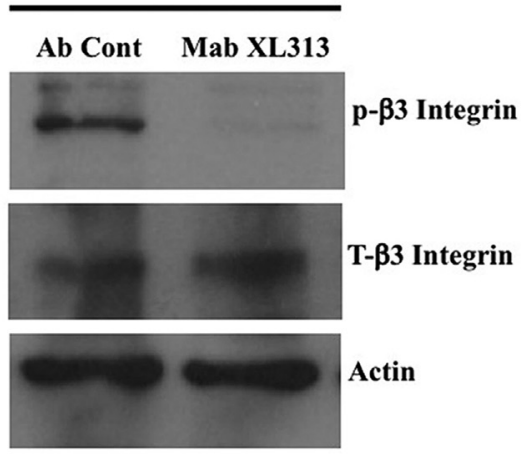

H

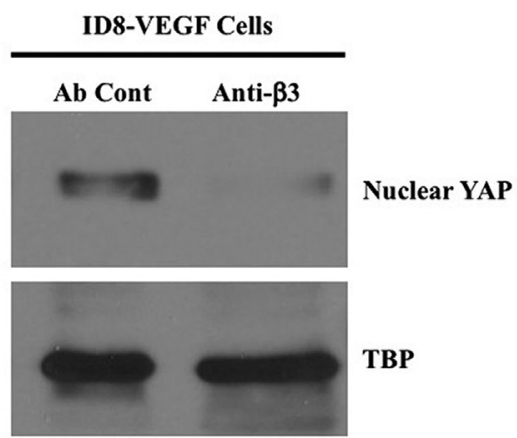

E

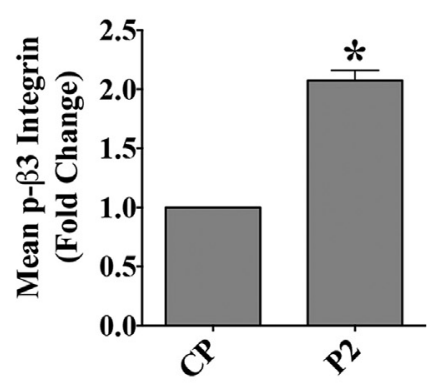

G

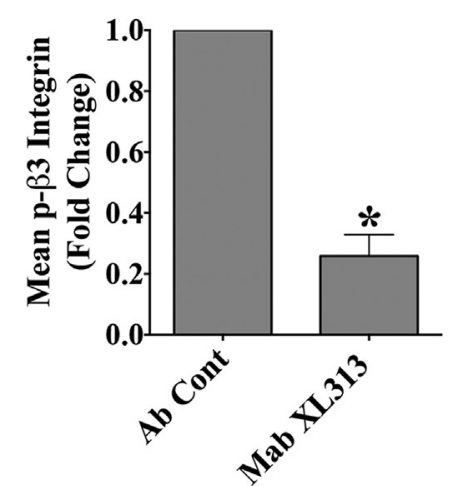

ID8-VEGF Cells

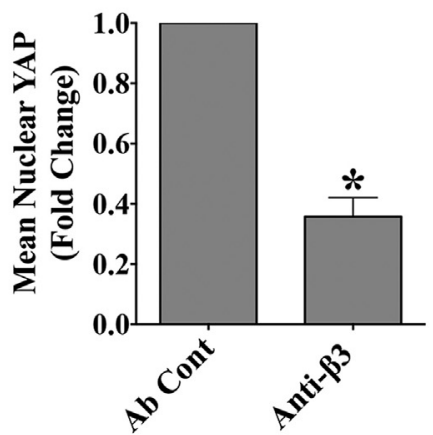

Figure 2 The RGDKGE-containing collagen fragment activates $\beta 3$ integrin and enhances the levels of nuclear yes-associated protein (YAP). A: Quantification of SKOV-3 cell binding to collagen peptide $\mathrm{P} 2$ in the presence of non-specific control antibody (NT), function-blocking anti- $\beta 3$-integrin antibody (Anti- $\beta 3$ ), or function-blocking anti- $\beta 1$-integrin antibody (Anti- $\beta 1$ ). B: Quantification of ID8-vascular endothelial growth factor (VEGF) cell binding to collagen peptide P2 in the presence of NT, function-blocking Anti- $\beta 3$, or function-blocking Anti- $\beta 1$. C: Western blot analysis of whole cell lysates of ID8-VEGF cells (left panels) and SKOV-3 cells (right panels) for the expression of integrin $\beta 3$ or actin. D: Whole cell lysates were prepared from ID8-VEGF cells stimulated with control peptide (CP) or collagen peptide P2 for $5 \mathrm{mi}$ nutes. Example of Western blot analysis for the levels of the phosphorylated $\beta 3$ integrin ( $p-\beta 3$ Integrin; Tyr 747), total $\beta 3$ integrin (T- $\beta 3$ Integrin), or loading control actin. E: Quantification of the mean fold change in phosphorylated $\beta 3$ integrin. F: Whole cell lysates were prepared from ID8-VEGF cells stimulated with collagen peptide P2 in presence of non-specific control antibody ( $A b$ Cont) and anti-RGDKGE collagen fragment antibody [monoclonal antibody (Mab) XL313]. Example of Western blot analysis for the levels of the $p-\beta 3$ Integrin), T- $\beta 3$ Integrin, or loading control actin. G: Quantification of the mean fold change in phosphorylated $\beta 3$ integrin. H: Nuclear fractions were prepared from ID8-VEGF cells stimulated with collagen peptide $P 2$ in presence of non-specific $A b$ Cont and Anti- $\beta 3$. Example of Western blot analysis for the levels of nuclear YAP or loading control TATA-binding protein (TBP). I: Quantification of the mean fold change in nuclear YAP. Data are expressed as means $\pm \operatorname{SEM}(\mathbf{A}, \mathbf{B}, \mathbf{E}, \mathbf{G}$, and $\mathbf{I}) . n \geq$ 3 independent experiments $(\mathbf{E}, \mathbf{G}$, and $\mathbf{I})$. * $P<0.05$. 
A

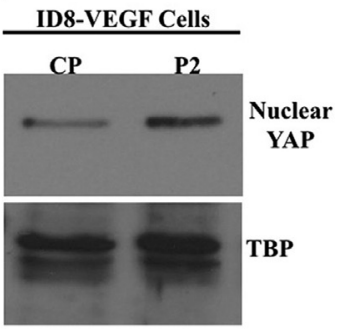

C

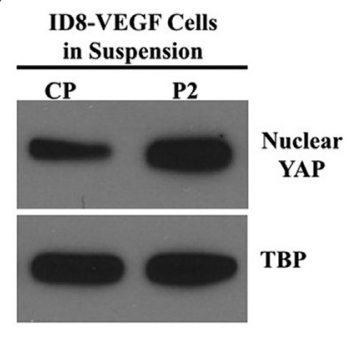

E

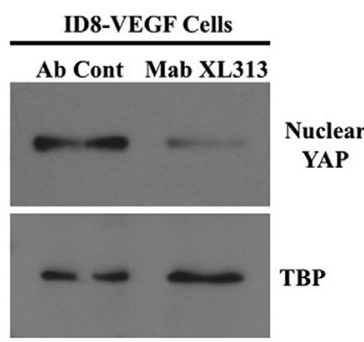

H

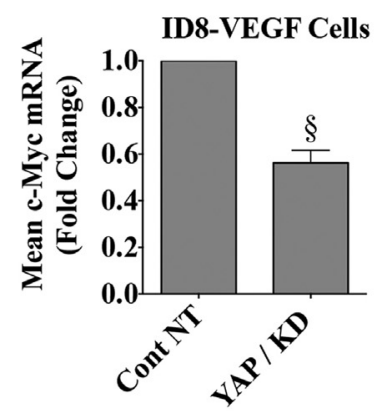

K

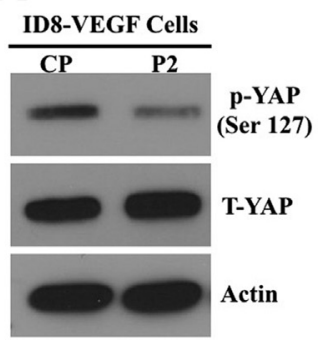

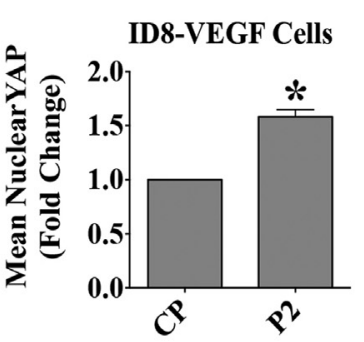

B
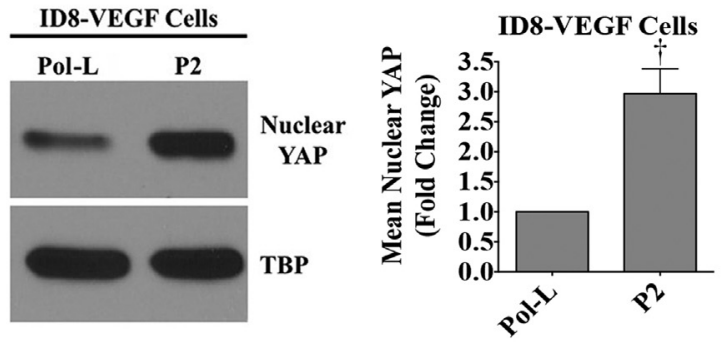

D
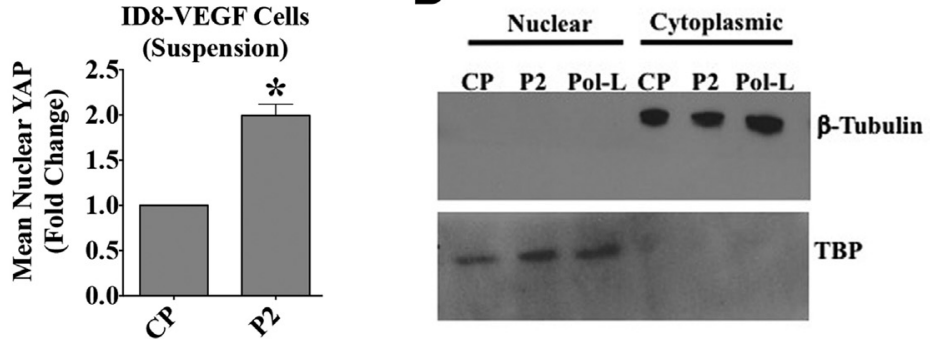

$\mathbf{F}$
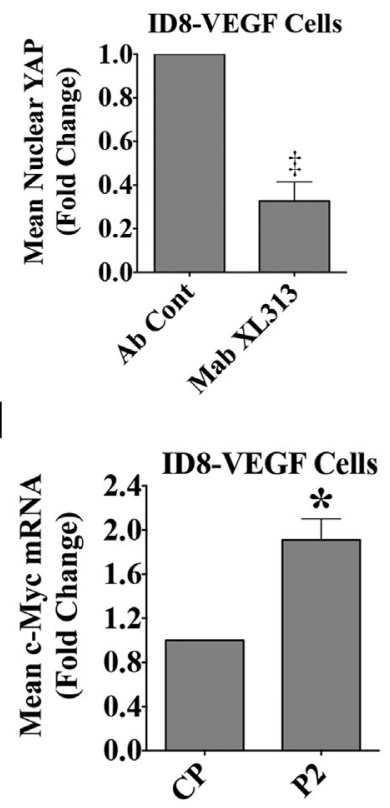

G

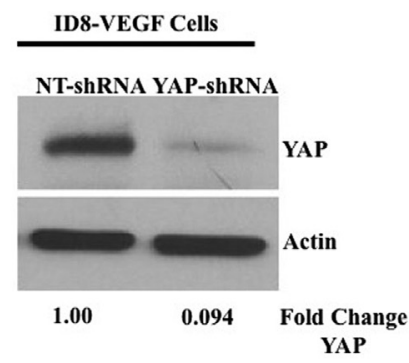

J

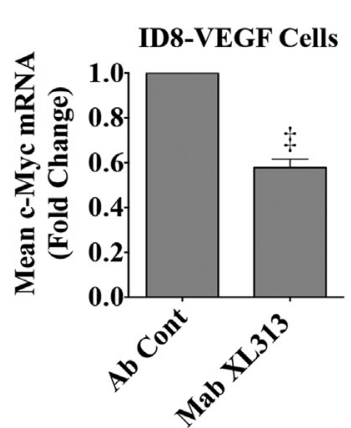

$\mathbf{L}$

M

N
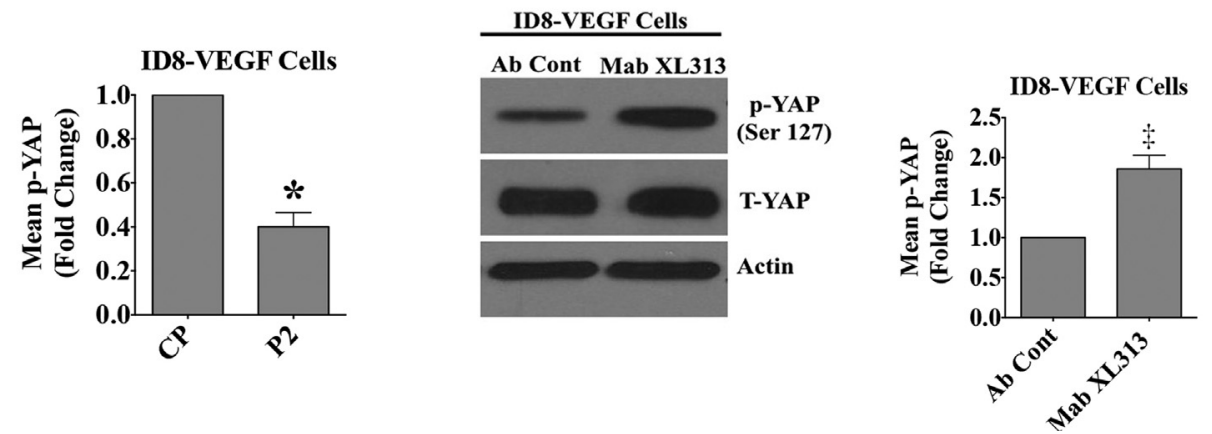
a function-blocking anti- $\beta 3$ integrin antibody had on nuclear YAP accumulation. Blocking P2 peptide cellular interactions with ID8-VEGF tumor cells by incubation with anti- $\beta 3$ integrin antibody significantly reduced the relative levels of nuclear YAP (Figure 2, H and I). Taken together, these data provide the first evidence that blocking $\beta 3$ integrin-mediated interactions with the RGDKGEcontaining collagen peptide P2 inhibits nuclear YAP accumulation in these ovarian carcinoma cells.

\section{The RGDKGE-Containing Collagen Fragment Regulates YAP Phosphorylation in Ovarian Tumor Cells}

Previous studies have implicated integrin signaling in regulating the activity and nuclear accumulation of YAP in different cell types. ${ }^{11,24-26}$ However, YAP can be controlled by diverse mechanisms, and depending on the particular cell type, may have opposing functions on tumor growth. ${ }^{16,18,19}$ Therefore, the effects of stimulation of ovarian tumor cells were examined in further detail with the RGDKGE collagen peptide P2 and its ability to alter nuclear accumulation of YAP. ID8-VEGF tumor cell binding to P2 resulted in significantly enhanced levels of nuclear localized YAP (Figure 3A). To provide evidence that the enhanced nuclear accumulation of YAP following binding to P2 was specific and not a result of simple mechanical adhesion, YAP nuclear accumulation was compared between cells binding to $\mathrm{P} 2$ or through nonintegrin interactions with poly-L-lysine. More important, poly-L-lysine supported ID8-VEGF cell binding similarly to P2 (Supplemental Figure S1A). Tumor cell binding to P2 significantly enhanced nuclear accumulation of YAP compared with cells binding to poly-L-lysine (Figure 3B). Similarly, stimulation of tumor cells in suspension with soluble P2 peptide also significantly enhanced accumulation of nuclear localized YAP compared with control (Figure 3C). More important, efficient nuclear and cytoplasmic separation of the samples under the indicated binding conditions was confirmed, as little, if any, $\beta$-tubulin was detected in nuclear fractions, but was clearly detected in cytoplasmic fractions, whereas TATA-binding protein was clearly detected in nuclear fractions, whereas little, if any, was observed in cytoplasmic fractions (Figure 3D). Finally, treatment with Mab XL313, but not control antibody, inhibited nuclear YAP levels (Figure 3, E and F). Nuclear localized YAP can interact with transcription factors, such as members of transcriptional enhancer factor domain family, to regulate protumorigenic gene expression programs that contribute to tumor growth and metastasis. ${ }^{27,28}$ Because YAP regulates the expression of protumorigenic target genes such as c-Myc, ${ }^{27-30}$ that are thought to play a role in ovarian cancer, it was examined whether YAP may play a role in regulating the expression of c-Myc in ID8-VEGF tumor cells. To facilitate these studies, YAP was knocked down in ID8-VEGF cells (Figure 3G) using a YAP-specific shRNA, and the relative levels of c-Myc mRNA were examined. Knocking down YAP significantly reduced the levels of c-Myc compared with control transfected cells, confirming the relevance of YAP in regulating c-Myc in these ovarian tumor cells (Figure 3H). Given our findings that cellular interactions with the RGDKGEcontaining collagen peptide $\mathrm{P} 2$ regulate YAP, the effects of P2 stimulation on the relative levels of c-Myc mRNA were examined by RT-qPCR. As expected, tumor cell interaction with the RGDKGE collagen fragment significantly enhanced c-Myc (Figure 3I), whereas blocking cellular engagement with the RGDKGE collagen fragment reduced c-Myc mRNA levels (Figure 3J). These data are

\footnotetext{
Figure 3 Regulation of yes-associated protein (YAP) phosphorylation and target gene c-Myc by the RGDKGE collagen fragment. A: Nuclear fractions were prepared from ID8-vascular endothelial growth factor (VEGF) cells stimulated with collagen peptide P2 or control peptide (CP). Left panels: Examples of Western blot analyses for the levels of nuclear YAP or loading control TATA-binding protein (TBP). Right panel: Quantification of the mean fold change in nuclear YAP. B: Nuclear fractions were prepared from ID8-VEGF cells binding to poly-L-lysine (Pol-L) or collagen peptide P2. Left panels: Examples of Western blot analyses for the levels of nuclear YAP or loading control TBP. Right panel: Quantification of the mean fold change in nuclear YAP. C: Nuclear fractions were prepared from ID8-VEGF cells binding to soluble collagen peptide P2 or CP in suspension. Left panels: Example of Western blot analyses for the levels of nuclear YAP or loading control TBP. Right panel: Quantification of the mean fold change in nuclear YAP. D: Western blot analysis of nuclear and cytoplasmic fractions prepared from ID8-VEGF cells stimulated with CP, collagen peptide P2, or poly-L-lysine for cytoplasmic marker $\beta$-tubulin or the nuclear marker TBP. E: Nuclear fractions were prepared from ID8-VEGF cells stimulated with collagen peptide P2 in the presence of non-specific control antibody (Ab Cont) or antiRGDKGE collagen fragment antibody [monoclonal antibody (Mab) XL313]. Example of Western blot analysis for the levels of nuclear YAP or loading control TBP. F: Quantification of the mean fold change in nuclear YAP from ID8-VEGF cells stimulated with collagen peptide P2 in the presence of non-specific Ab Cont or anti-RGDKGE collagen fragment antibody (Mab XL313). G: Western blot analysis of whole cell lysates prepared from control shRNA transfected (NT-shRNA) and Yap-shRNA transfected ID8-VEGF ovarian tumor cells for YAP expression or loading control actin. Relative fold change in levels of protein expression was determined by ImageJ software version 5.0. H: ID8-VEGF cells were transfected with either a nontargeting control shRNA (Cont-NT) or YAP-specific shRNA (YAP/KD). Quantification of the mean relative fold change in c-Myc mRNA. I: Quantification of the mean fold change in c-Myc mRNA levels in ID8-VEGF cells stimulated with CP or collagen peptide P2. J: Quantification of the mean fold change in c-Myc mRNA levels in ID8-VEGF cells stimulated with collagen peptide P2 in the presence of non-specific Ab Cont or anti-RGDKGE collagen fragment antibody (Mab XL313). K: Western blot analysis of cytosolic fractions prepared from ID8-VEGF cells stimulated with CP or collagen peptide P2 for the levels of phosphorylated YAP ( $p$-YAP; Ser-127), total YAP (T-YAP), or loading control actin. L: Quantification of the mean fold change in phosphorylated YAP from ID8-VEGF cells stimulated with CP or collagen peptide P2. M: Western blot analysis of cytosolic fractions prepared from ID8-VEGF cells stimulated with collagen peptide P2 in the presence of non-specific Ab Cont or anti-RGDKGE collagen fragment antibody (Mab XL313) for p-YAP, T-YAP, or loading control actin. N: Quantification of the mean fold change in p-YAP from ID8-VEGF cells stimulated with collagen peptide $\mathrm{P} 2$ in the presence of non-specific Ab Cont or anti-RGDKGE collagen fragment antibody (Mab XL313). Data are expressed as means \pm SEM (A-C, F, H-J, L, and $\mathbf{N}) . n \geq 3$ independent experiments (A, bottom panel, $\mathbf{F}, \mathbf{I}, \mathbf{J}, \mathbf{L}$, and $\mathbf{N}$ ); $n=3$ independent experiments (B and $\mathbf{C}$, bottom panels); $n=4$ independent experiments $(\mathbf{H})$. ${ }^{*} P<0.05$ versus $\mathrm{CP} ;{ }^{\dagger} P<0.05$ versus Pol-L; ${ }^{\ddagger} P<0.05$ versus Ab Cont; ${ }^{\S} P<0.05$ versus Cont NT.
} 
A

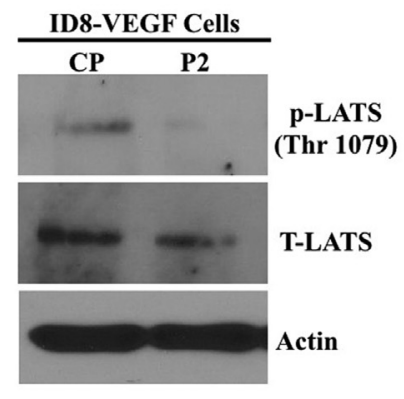

E
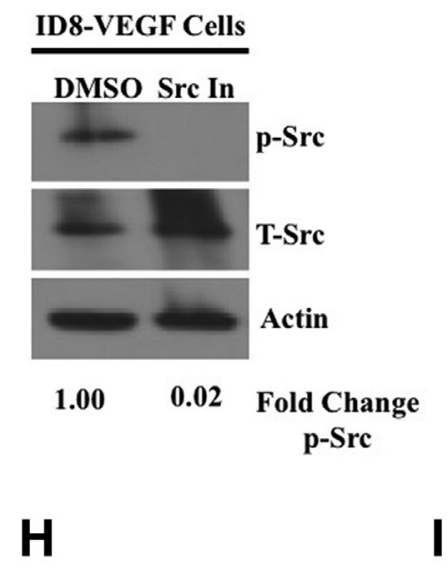

ID8-VEGF Cells (Src In)

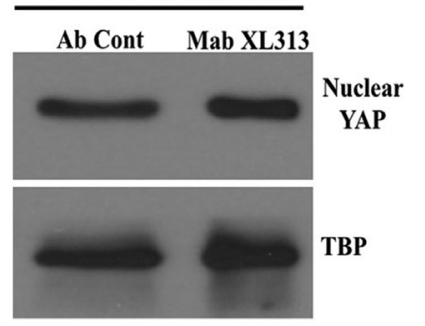

L

ID8-VEGF Cells (Scr In) Ab Cont Mab XL313
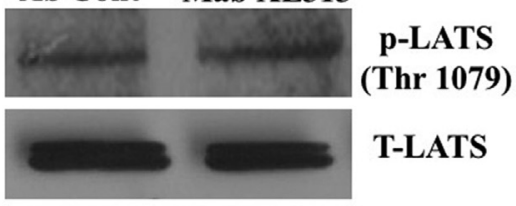

T-LATS

Actin
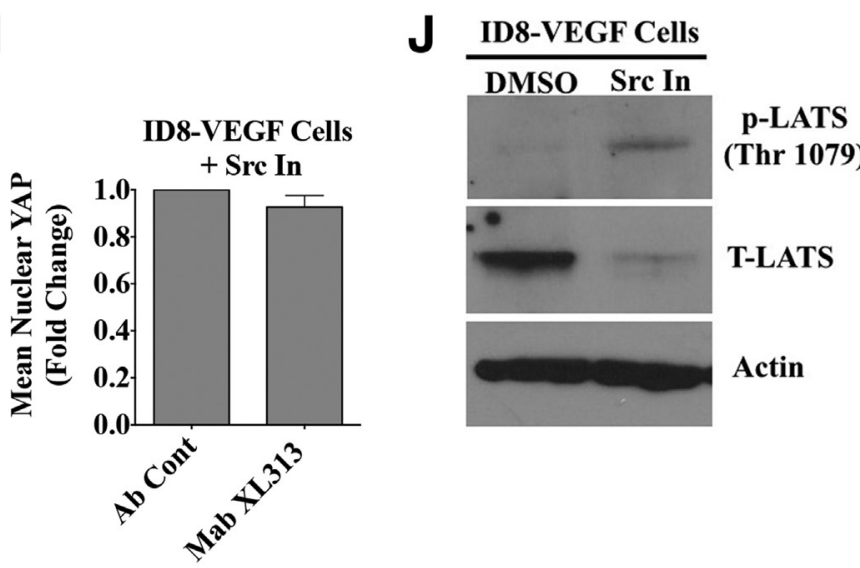

(Thr 1079)

T-LATS
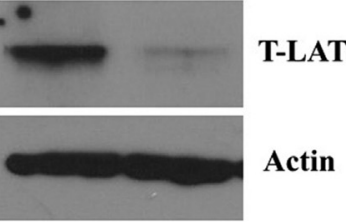

G

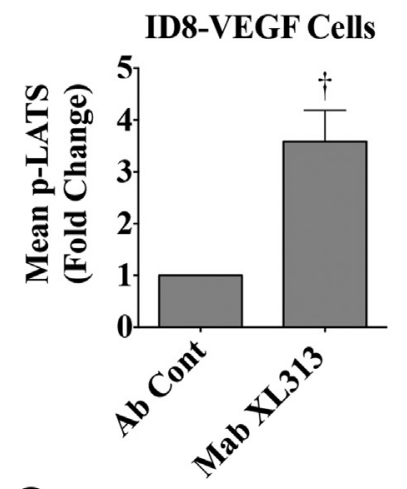

ID8-VEGF Cells

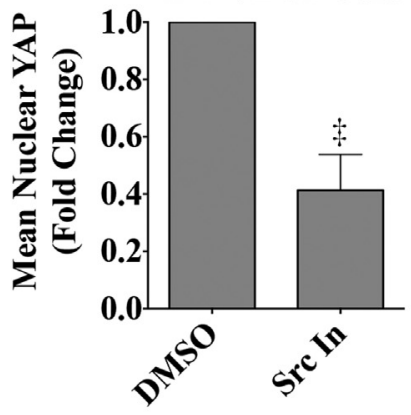

K

ID8-VEGF Cells

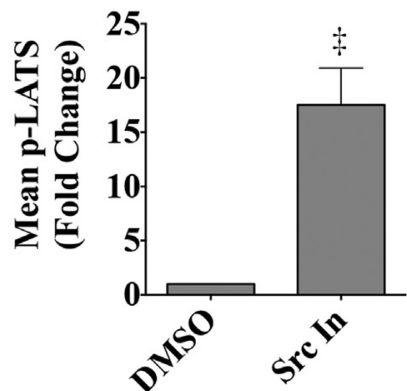

$\mathbf{N}$
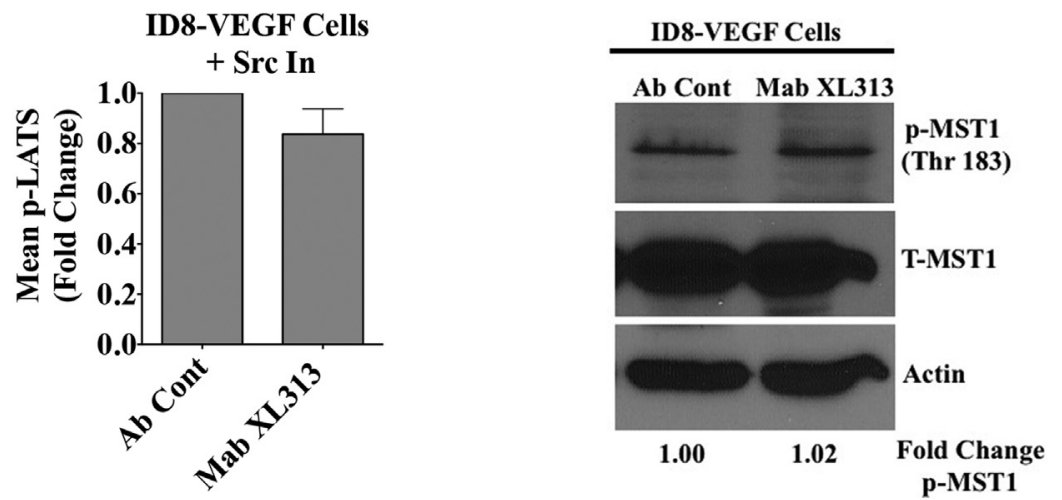
consistent with the ability of RGDKGE collagen fragment to modulate the expression of a known YAP target gene.

The differential localization of YAP between the cytoplasm and the nucleus, where it can function as a transcriptional co-activator regulating gene expression programs, is thought to be controlled by multiple mechanisms, including hippo-dependent and hippo-independent signaling events. ${ }^{16,20,21}$ To uncover possible mechanisms that might contribute to the ability of this collagen peptide to enhance YAP nuclear localization, YAP phosphorylation was studied, because the phosphorylation of YAP on serine127 has been shown to play a role in limiting the relative levels of active YAP within the nucleus. ${ }^{20,21}$ Interestingly, ID8-VEGF cell interactions with P2 collagen peptide significantly inhibited the levels of YAP serine 127 phosphorylation (Figure 3, K and L), whereas blocking cellular interactions with $\mathrm{P} 2$ enhanced YAP phosphorylation (Figure 3, M and N). Collectively, these findings suggest that cellular interactions with the RGDKGE collagen fragment can enhance nuclear accumulation of YAP in ovarian carcinoma cells and that this differential accumulation of nuclear YAP was associated with alterations in YAP phosphorylation and expression of the YAP target gene cMyc.

\section{The RGDKGE-Containing Collagen Fragment Regulates the Hippo Pathway Effector Kinase LATS1 by an Src-Dependent Mechanism}

As mentioned above, the nuclear accumulation of YAP is thought to be governed by multiple mechanisms. ${ }^{16,20,21}$ LATS1 is a well-established effector molecule of the hippo signaling cascade known to phosphorylate YAP, leading to inactivation and reduced nuclear accumulation. ${ }^{20,21,31,32}$
The levels of phosphorylated LATS1 in ID8-VEGF tumor cells were examined following stimulation with $\mathrm{P} 2$ collagen peptide. Stimulation of tumor cells with $\mathrm{P} 2$ collagen peptide significantly reduced the relative levels of phosphorylated LATS1 (Figure 4, A and B), whereas similar experiments performed in the presence of Mab XL313 demonstrated a significant enhancement of levels of phosphorylated LATS1 on Thr-1079 (>threefold) following blocking cellular interactions with P2 collagen peptide (Figure 4, C and D). These data are consistent with the ability of Mab XL313 to inhibit nuclear accumulation of YAP that is associated with the regulation of LATS1.

Given that both LATS1 and YAP have been suggested to be controlled in part by Src family kinases, ${ }^{33-35}$ and that $\mathrm{Src}$ is a major downstream effector molecule that can be regulated by integrin signaling, it was examined whether $\mathrm{Src}$ plays a role in the ability of the P2 collagen fragment to control YAP nuclear accumulation. The relative levels of P2-stimulated nuclear YAP was exmianed in tumor cells in which Src was inhibited. Briefly, ID8-VEGF cells were first pre-incubated with the Src inhibitor dasatinib to confirm that it could block Src activity in these cells (Figure 4E), cells were stimulated with $\mathrm{P} 2$, and the relative levels of nuclear YAP were quantified. Inhibiting Src reduced the relative levels of P2 peptide-stimulated nuclear YAP accumulation compared with control (Figure 4, F and G). Moreover, inhibiting Src also prevented the ability of Mab XL313 to alter nuclear accumulation of YAP, as Mab XL313 failed to significantly reduce the levels of nuclear YAP in Srcinhibited cells (Figure 4, H and I). Finally, the effects of blocking Src on LATS phosphorylation were studied. Interestingly, although blocking Src in these ID8-VEGF cells significantly enhanced the relative levels of LATS phosphorylation compared with control (Figure 4, J and K),

\footnotetext{
Figure 4 Regulation of large tumor suppressor kinase-1 (LATS1) phosphorylation by the RGDKGE collagen fragment. A: Cytosolic fractions were prepared from ID8-vascular endothelial growth factor (VEGF) cells stimulated with collagen peptide P2 or control peptide (CP). Example of Western blot analysis for the levels of phosphorylated LATS1 (p-LATS1; Thr 1079) or loading control actin. B: Quantification of the mean fold change in p-LATS1 from ID8-VEGF cells stimulated with collagen peptide P2 or CP. C: Cytosolic fractions were prepared from ID8-VEGF cells stimulated with collagen peptide P2 in the presence of nonspecific control antibody (Ab Cont) or anti-RGDKGE collagen fragment antibody [monoclonal antibody (Mab) XL313]. Example of Western blot analysis for the level of p-LATS1 or loading control actin. D: Quantification of the mean fold change in p-LATS1 from ID8-VEGF cells stimulated with collagen peptide P2 in the presence of non-specific Ab Cont or anti-RGDKGE collagen fragment antibody (Mab XL313). E: ID8-VEGF cells were treated (1 $\mu$ mol/L) with the Src inhibitor dasatinib (Src In) or control [dimethyl sulfoxide (DMSO)]. Representative example of Western blot analysis for phosphorylated Src ( $p$-Src; Tyr 416), total Src (T-Src), or loading control actin. Relative fold change in levels of protein expression was determined by ImageJ software version 5.0. F: Nuclear fractions were prepared from ID8-VEGF cells stimulated with collagen peptide P2 in the presence of control (DMS0) or Src In. Example of Western blot analysis for the levels of nuclear yes-associated protein (YAP) or loading control TATA-binding protein (TBP). G: Quantification of the mean fold change in nuclear YAP from ID8-VEGF cells stimulated with collagen peptide P2 in the presence of control (DMSO) or Src In. H: Western blot analysis of nuclear fractions from ID8-VEGF cells stimulated with collagen peptide P2 in the presence of the Src In and in the presence of non-specific Ab Cont or anti-RGDKGE collagen fragment antibody (Mab XL313) for the levels of nuclear YAP or loading control TBP. I: Quantification of the mean fold change in nuclear YAP from nuclear fractions from ID8-VEGF cells stimulated with collagen peptide P2 in the presence of the Src In and in the presence of non-specific Ab Cont or anti-RGDKGE collagen fragment antibody (Mab XL313). J: Western blot analysis of ID8-VEGF cells treated with DMS0 or Src In for p-LATS1 or loading control actin. K: Quantification of the mean fold change in p-LATS1 from ID8-VEGF cells treated with DMS0 or Src In. L: Western blot analysis of cytosolic fractions from ID8-VEGF cells stimulated with collagen peptide P2 in the presence of the Src In and the presence of non-specific Ab Cont or anti-RGDKGE collagen fragment antibody (Mab XL313) for p-LATS1 or loading control actin. M: Quantification of the mean fold change in p-LATS1 from ID8-VEGF cells stimulated with collagen peptide P2 in the presence of the Src In and the presence of non-specific Ab Cont or anti-RGDKGE collagen fragment antibody (Mab XL313). N: Western blot analysis of ID8-VEGF cells incubated in the presence Mab XL313 or non-specific control antibody seeded on RGDKGE-containing collagen peptide P2 for phosphorylated MST1 (p-MST1; Thr 183), total MST1 (T-MST1), or loading control actin. Relative fold change in levels of protein expression was determined by ImageJ software. Data are expressed as means \pm SEM (B, D, G, I, K, and M). $n \geq 3$ independent experiments (B, D, G, I, K, and $\mathbf{M})$. ${ }^{\star} P<0.05$ versus $C P$; ${ }^{\dagger} P<0.05$ versus Ab Cont; ${ }^{\ddagger} P<0.05$ versus DMSO. T-LATS1, total LATS1.
} 
A

SKOV-3 Ovarian Carcinoma

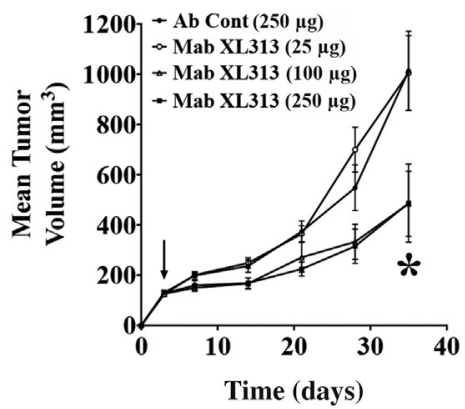

E
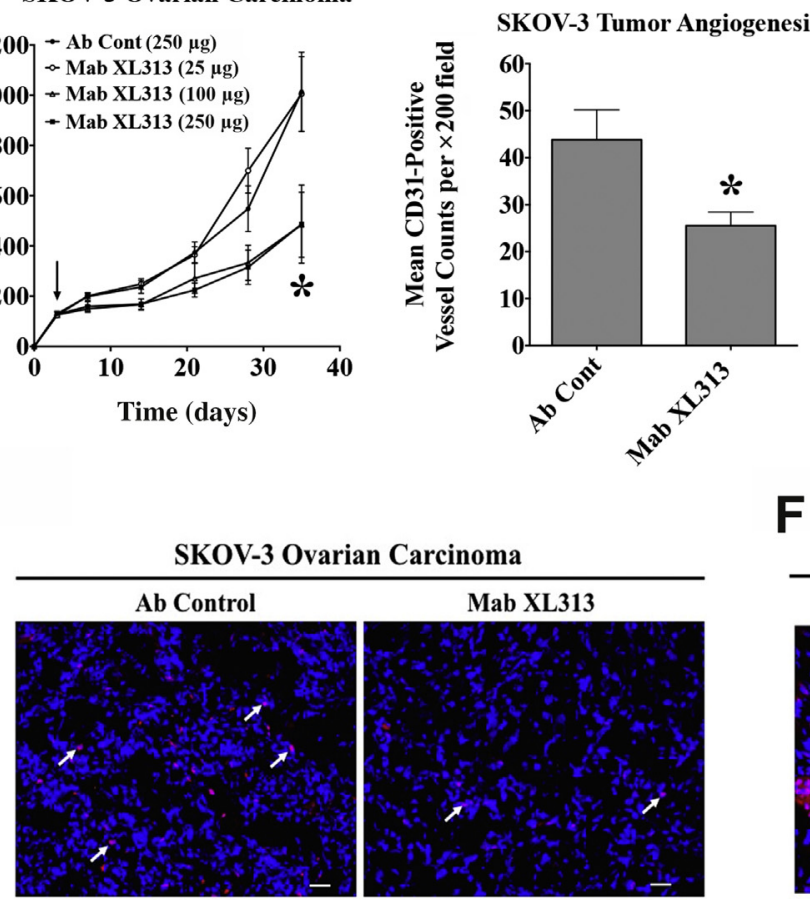

C

S.C. ID8-VEGF Ovarian Tumor

D
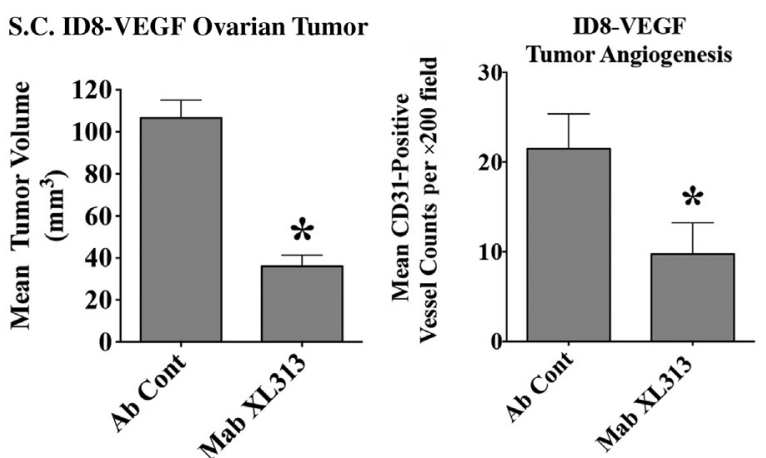

SKOV-3 Ovarian Carcinoma

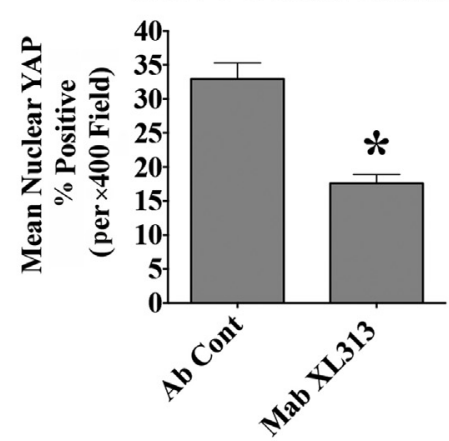

G

ID8-VEGF Tumor Ascites

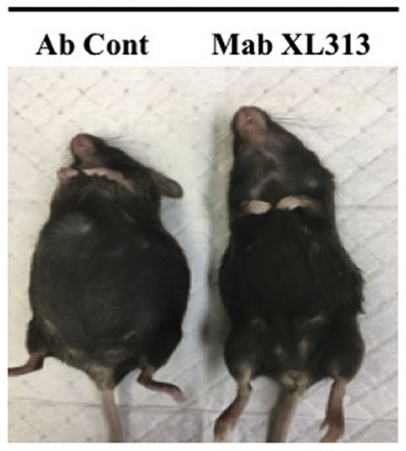

ID8-VEGF

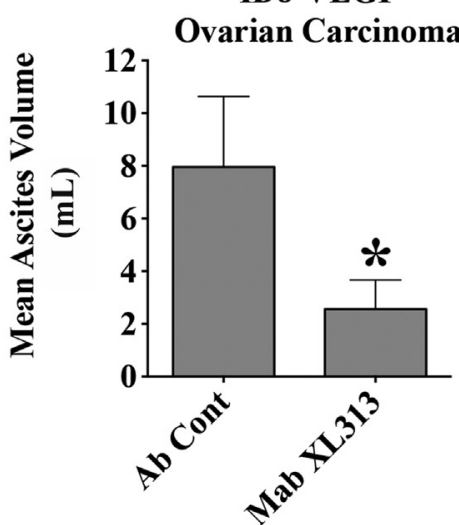

ID8-VEGF Ovarian Carcinoma

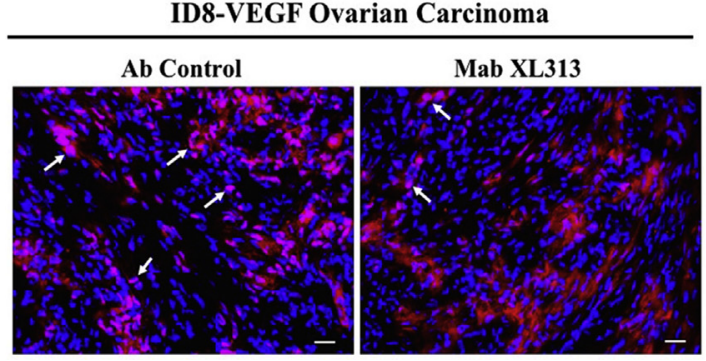

ID8-VEGF Ovarian Carcinoma

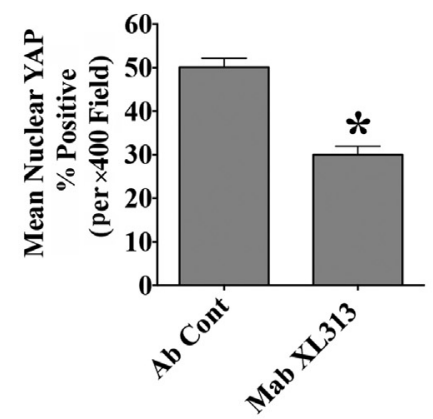

H

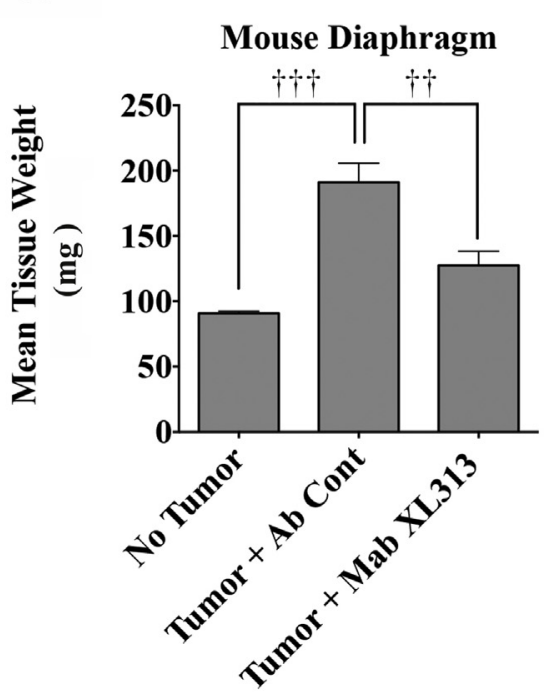


surprisingly, this treatment also reduced the overall levels of LATS (Figure 4J). Although the mechanism by which Src reduced the overall levels of LATS, and enhanced the levels of the phosphorylated LATS in these ID8-VEGF cells, is currently not known, our data indicate an important role for Src in regulating LATS under these experimental conditions.

Given that blocking cellular interactions with the P2 collagen peptide significantly enhanced phosphorylated LATS1, the effects of Src inhibition on the ability of Mab XL313 to control LATS1 phosphorylation were studied. As expected, blocking Src inhibited the ability of Mab XL313 to regulate LATS1 phosphorylation (Figure 4, L and M). Interestingly, blocking cellular interactions with $\mathrm{P} 2$ had no detectable effect on the relative levels of activated hippo kinase MST1 (Figure 4N). Taken together, these findings suggest that the ability of the RGDKGE collagen fragment to regulate the hippo effector kinase LATS1 and its downstream target YAP in these tumor cells depends on Src.

\section{Selective Targeting of the RGDKGE-Containing Collagen Fragment Inhibits Ovarian Tumor Growth}

The RGDKGE collagen fragment was previously shown to play a role in angiogenesis and inflammation. ${ }^{11}$ However, it is not known whether this collagen fragment may impact tumor growth in vivo. It was studied whether selective targeting of this collagen fragment might regulate ovarian tumor growth. The dose-dependent effects of Mab XL313 on ovarian tumors growing in mice were studied. Human SKOV-3 ovarian tumor cells were injected subcutaneously. Once tumors reached a size of approximately $100 \mathrm{~mm}^{3}$, mice were treated with Mab XL313 or non-specific control antibody three times per week at the doses indicated. Treatment with Mab XL313 exhibited dose-dependent inhibitory activity, and showed a significant inhibition (approximately $50 \%$ ) on day 35 (Figure 5A). Given our previous studies indicating that selectively targeting the RGDKGE collagen fragment could inhibit growth factor-induced angiogenesis in the chick embryo model, ${ }^{11}$ SKOV-3 tumors were examined for the relative levels of angiogenesis by quantifying the number of CD31-expressing tumor vessels. Tumors from mice treated with Mab XL313 resulted in significantly reduced levels of tumor vessels (approximately 35\%) (Figure 5B). To confirm the antitumor activity of Mab XL313 in a second ovarian tumor model, the effects of Mab XL313 on ID8-VEGF tumor growth were studied. Murine ID8-VEGF tumor cells were injected subcutaneously, and beginning on day 3 , mice were treated as before. Although these s.c. ID8-VEGF tumors grew slowly and showed evidence of regression irrespective of the treatment, the size of ID8-VEGF tumors from mice treated with Mab XL313 was significantly reduced by approximately $50 \%$ compared with control (Figure 5C). Interestingly, incubation of ID8-VEGF cells with Mab XL313 had no significant inhibitory activity on cell growth in vitro compared with control antibody (Supplemental Figure S1B). However, similar to that observed in SKOV-3 tumors, Mab XL313 significantly inhibited tumor angiogenesis in ID8-VEGF tumors (Figure 5D). Given our studies indicating that Mab XL313 could reduce the levels of nuclear accumulation of YAP in ovarian tumor cells in vitro, ovarian tumors from mice treated with Mab XL313 were examined for the relative levels of nuclear localized YAP. Although clear variation in the overall levels of YAP was observed between SKOV-3 and ID8-VEGF tumors (Figure 5, E and F), the relative levels of nuclear localized YAP were consistently reduced within tumors from mice treated with Mab XL313 compared with control (Figure 5, E and F).

Ovarian tumors growing within the s.c. space may not represent an optimal model to study ovarian tumor growth, due in part to the lack of a mesothelial cell layer covering tissues that are typically colonized within the peritoneal cavity, where ovarian tumors typically grow. ${ }^{36,37}$ Therefore, the effects of targeting the RGDKGE collagen fragment on ID8-VEGF ascites fluid formation and tumor colonization of the diaphragm, which represent a common tissue location of

\footnotetext{
Figure 5 Inhibition of the RGDKGE collagen fragment inhibits ovarian tumor growth. A: Quantification of the dose-dependent effects of anti-RGDKGE collagen fragment antibody [monoclonal antibody (Mab) XL313] or non-specific control antibody (Ab Cont) on the growth of SKOV-3 tumors in vivo. Arrow indicates start of treatment with antibodies. Data points indicate tumor volumes. B: Quantification of the effects of anti-RGDKGE collagen fragment antibody (Mab XL313) or non-specific Ab Cont on SKOV-3 tumor angiogenesis. Data bars indicate number of CD31-positive vessels per $\times 200$ field within five independent fields from five different tumors per condition. C: Quantification of the effects of anti-RGDKGE collagen fragment antibody (Mab XL313) or nonspecific Ab Cont on the s.c. growth of ID8-vascular endothelial growth factor (VEGF) tumors in vivo. Data bars indicate tumor volume measured on day 42. D: Quantification of the effects of anti-RGDKGE collagen fragment antibody (Mab XL313) or non-specific Ab Cont on ID8-VEGF tumor angiogenesis. Data bars indicate number of $\mathrm{CD} 31$-positive vessels per $\times 200$ field within eight independent fields from five to six different tumors per condition. E: Tissue sections from SKOV-3 tumors from mice treated with control antibody or Mab XL313 were analyzed for the relative levels of nuclear localized yes-associated protein (YAP). Top panels: Examples of nuclear localized YAP; arrows indicate examples of nuclear accumulation of YAP (pink indicates overlap of blue DAPI and red YAP staining). Bottom panel: Quantification of mean percentage nuclear-positive cells per $\times 400$ field from seven to nine fields from four independent tumors per condition. F: Tissue sections from ID8-VEGF tumors from mice treated with control antibody or Mab XL313 were analyzed for the relative levels of nuclear localized YAP. Top panels: Examples of nuclear localized YAP; arrows indicate examples of nuclear accumulation of YAP (pink indicates overlap of blue DAPI and red YAP staining). Bottom panel: Quantification of mean percentage nuclear-positive cells per $\times 400$ field from four to seven fields from five independent tumors per condition. G: Left panel: Representative example of the differential abdominal distension of ID8-VEGF cell-injected mice treated for 13 weeks with anti-RGDKGE collagen fragment antibody (Mab XL313) or non-specific Ab Cont. Right panel: Quantification of the effects of anti-RGDKGE collagen fragment antibody (Mab XL313) or non-specific Ab Cont on the relative volume of ascites. Data bars indicate ascites fluid volume measured at 13 weeks. H: Quantification of the effects of anti-RGDKGE collagen fragment antibody (Mab XL313) or non-specific Ab Cont on the wet weights of mouse diaphragms. Data bars indicate wet weight measured at 13 weeks. Data are expressed as means \pm SEM $(\mathbf{A}-\mathbf{H}) . n=8$ per group $(\mathbf{A}$ and $\mathbf{C}) ; n=5$ per group $(\mathbf{G}$ and $\mathbf{H})$. ${ }^{\star} P<0.05$ versus $\mathrm{Ab}$ Cont; ${ }^{\dagger \dagger} P<0.01$, and ${ }^{\dagger \dagger} P<0.001$. Scale bars $=20 \mu \mathrm{m}$ (E and $\left.\mathbf{F}\right)$.
} 
ovarian tumors in mice and humans, were studied. ${ }^{36,37}$ To this end, mice were injected intraperitoneally with ID8VEGF cells, which were allowed to grow for 4 weeks before initiating treatment. Beginning at 4 weeks, mice were treated with either non-specific control antibody or Mab XL313 two times per week. At the end of 13 weeks, mice were sacrificed and ascites fluid and diaphragms were examined. Mice treated with control antibody formed ascites, as indicated by extensive distention of the abdominal cavities, whereas mice treated with Mab XL313 exhibited less abdominal distension (Figure 5G). Quantification of the relative volume of tumor ascites fluid indicated that mice treated with Mab XL313 had significantly reduced levels by $>60 \%$ (Figure 5G). Moreover, quantification of tumor burden, as indicated by wet tissue weights of the diaphragms, indicated that mice treated with Mab XL313 exhibited an approximately $30 \%$ reduction in wet weight compared with controls (Figure $5 \mathrm{H}$ ). These novel findings suggest that the endogenously generated RGDKGE collagen fragment plays a role in ovarian tumor growth in vivo.

\section{Mab XL313-Mediated Inhibition of Ovarian Tumor Growth Depends on Altering YAP}

The transcriptional co-activator YAP plays roles in angiogenesis, tumor growth, and metastasis in multiple tumor models. ${ }^{16,24-28}$ However, other studies have suggested that YAP may also have tumor suppressive functions, depending on the tissue location and cell type. ${ }^{18,19}$ Our current studies indicate that the RGDKGE-containing collagen fragment can bind to integrin $\beta 3$ and stimulate a signaling cascade, leading to enhanced nuclear accumulation of YAP. It was next determined whether the antitumor activity of Mab XL313 depends on altering YAP. To facilitate these studies, the expression of YAP was knocked down in SKOV-3 cells using a YAP-specific shRNA (Figure 6A), and examined the antitumor activity of Mab XL313 on the growth of either control transfected SKOV-3 cells (NT-SKOV-3) or SKOV3 cells in which YAP was knocked down (YAP-KD-SKOV$3)$. Tumor cell variants were injected in mice, and beginning on day 3 , mice were treated three times per week with 100 $\mu \mathrm{g}$ of Mab XL313 or control antibody. Mab XL313 significantly inhibited the growth of control transfected (NT-SKOV-3) tumors by approximately $50 \%$ on day 35 (Figure 6B). In contrast, Mab XL313 failed to significantly inhibit the growth of SKOV-3 tumors (YAP-KD-SKOV-3) in which the levels of YAP had been reduced (Figure 6C). These data are consistent with notion that the antitumorigenic activity of Mab XL313 depends on inhibiting YAP. Collectively, our data are consistent with a working model in which ovarian tumor cell interactions with an endogenously generated RGDKGE-containing collagen fragment stimulate a $\beta 3$-integrin signaling cascade that results in suppressing phosphorylation of the effecter kinase LATS1, ultimately leading to enhanced nuclear accumulation of YAP (Figure 6D).
A

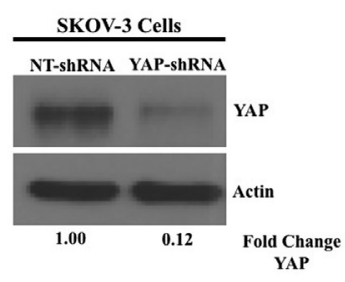

C

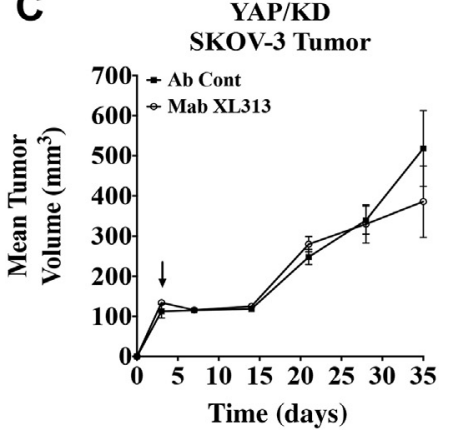

B

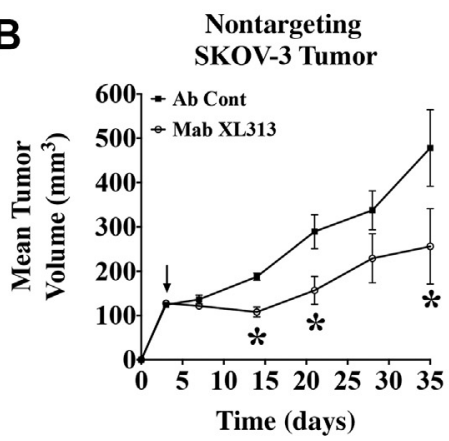

D

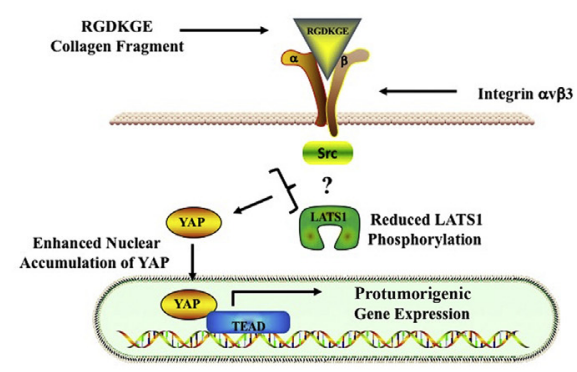

Figure 6 Monoclonal antibody (Mab) XL313 -mediated inhibition of ovarian tumor growth depends on yes-associated protein (YAP). A: Western blot analysis of whole cell lysates prepared from control shRNA-transfected (NT-shRNA) and $Y A P$-shRNA-transfected SKOV-3 ovarian tumor cells for YAP or loading control actin. Relative fold change in levels of protein expression was determined by ImageJ software version 5.0. B: Quantification of the effects of anti-RGDKGE collagen fragment antibody (Mab XL313) or non-specific control antibody (Ab Cont) on the growth of control-transfected SKOV-3 tumor cells (nontargeting SKOV-3) in vivo. Arrow indicates start of treatment with antibodies. Data points indicate tumor volumes. C: Quantification of the effects of anti-RGDKGE collagen fragment antibody (Mab XL313) or non-specific Ab Cont on the growth of SKOV-3 tumor cells in which YAP levels have been knocked down (YAP/KD-SKOV-3). Arrow indicates start of treatment with antibodies. Data points indicate tumor volumes. D: Working model of a potential mechanism by which the endogenously generated RGDKGE collagen fragment may regulate the nuclear accumulation of YAP. Data are expressed as means \pm SEM (B and $\mathbf{C}$ ). $n=8$ per group (B and $\mathbf{C}$ ). ${ }^{*} P<0.05$ versus $A b$ Cont at each time point. LATS1, large tumor suppressor kinase1; TEAD, transcriptional enhancer factor domain. 


\section{Discussion}

Although the ability of the local stromal microenvironment to control tumor growth has been appreciated for years, effectively translating this cellular and molecular insight into new therapeutic strategies has been slow to emerge, due in part to the complexity of the various functional elements that constitute the tumor microenvironment. Stromal cells known to influence tumor growth include endothelial cells and a variety of differentially polarized fibroblasts and immune cells. ${ }^{1-3}$ These diverse groups of cells not only modulate the behavior of solid tumors by secreting bioactive molecules that can alter the structural integrity of the ECM, but also secrete many soluble factors that directly bind to nearby tumor cells and modulate diverse signaling pathways. ${ }^{1-6,37,38}$ In a reciprocal manner, stromal cells can also respond to soluble factors released from tumor cells, and these soluble cell-cell communication pathways help support tumor progression. ${ }^{1-6,37,38}$ This complex cross talk between different cell types and between cells and a variety of ECM components plays critical roles in regulating tumor growth and metastasis, as well as influencing how tumors respond to different cancer therapies. ${ }^{1-6,37,38}$ Identifying soluble bioactive molecules that have the capacity to alter the behavior of both stromal and tumor cells will likely provide important new insight into how cross-talk signaling mechanisms are coordinated, and may help with the design of more effective therapeutic strategies to manage malignant tumor progression.

We previously identified a $16-\mathrm{kDa}$ RGDKGE-containing collagen fragment that was actively secreted by M2-like macrophages and stimulated nuclear accumulation of YAP in endothelial cells. ${ }^{11}$ Although it is well documented that YAP can differentially regulate several distinct gene expression programs in different cell types, the role of YAP in controlling biological processes such as organ size, stem cell functions, differentiation, and tumor growth, likely depends on how YAP activity is controlled in distinct cell populations within a given tissue microenvironment. ${ }^{39-41} \mathrm{In}$ fact, the precise biological impact of YAP, as it relates to both normal physiological processes as well as diseases such as cancer, has been shown to be context-dependent. $^{39-41}$ For example, YAP has been shown to control the size of organs such as the heart and liver, ${ }^{42-44}$ whereas loss of YAP either had a minimal effect or reduced the size of other histologically distinct organs such as the intestine and pancreas. ${ }^{45-47}$ Moreover, although reducing YAP activity inhibits the growth of tumors such as melanoma and lung carcinomas, ${ }^{48,49}$ other studies suggest that under specific circumstances, YAP may have tumor suppressive functions in breast and liver carcinomas. ${ }^{18,19}$ These and other studies indicate the need for a more in-depth understanding of the different mechanisms by which YAP is regulated within distinct cell types.

Uncovering novel mechanisms by which YAP may be regulated under pathologic conditions may allow for more optimized strategies to control YAP activity in the context of tumor growth. YAP activity can be regulated by diverse mechanisms, including cell adhesion, mechanical forces, stress signaling, and soluble factors, ${ }^{16,20,21}$ some of which can be altered by the canonical hippo signaling cascade. ${ }^{16,20,21}$ In addition, YAP can also be regulated by hippo pathway-independent mechanisms, such as those involved in altered actin polymerization. ${ }^{16,20,21}$ These, coupled with the known functions of YAP in controlling gene expression programs, ${ }^{27,28}$ defining strategies to selectively control YAP activity under pathologic conditions will likely contribute to optimizing the therapeutic efficacy of targeting YAP.

Although intact collagen within the insoluble ECM is thought to regulate ovarian carcinoma progression, ${ }^{36,50}$ little is known as to whether soluble bioactive collagen fragments regulate ovarian tumor growth in vivo. Herein, we provide evidence, for the first time, that a unique RGDKGEcontaining collagen fragment can be detected in ovarian tumors growing in mice and can also be generated by some ovarian tumor cell lines. Interestingly, although variable levels of the RGDKGE-containing collagen fragment were detected in some normal skin samples from mice, little, if any, of this collagen fragment was detected in normal peritoneal tissues, where ovarian tumors often grow. Given our previous studies ${ }^{11}$ and our current data, we sought to determine whether the RGDKGE collagen fragment might also directly bind to ovarian carcinoma cells and regulate tumor growth. Our data indicate that SKOV-3 and ID8VEGF ovarian tumor cells can bind to the soluble RGDKGE collagen fragment using $\beta 3$ integrin as a function-blocking antibody directed to this integrin inhibited binding, whereas a function-blocking antibody directed to $\beta 1$-containing integrins failed to demonstrate any effect. Ovarian tumor cell binding to the RGDKGE collagen peptide led to enhanced phosphorylation and activation of $\beta 3$ integrin, and enhanced nuclear accumulation of YAP, which could be specifically inhibited by directly targeting the RGDKGE collagen peptide with Mab XL313. More important, the exact molecular mechanism by which Src controls the ability of the RGDKGE collagen fragment to regulate LATS1 and YAP is not completely understood, and work is currently underway to define this signaling pathway.

The precise role of $\beta 3$ integrin in controlling tumor growth is complex, as studies have provided evidence for both protumorigenic as well as tumor suppressive functions. For example, some studies have shown correlations between high expression of $\beta 3$ integrin and poor prognosis in patients with melanoma and breast carcinoma. ${ }^{51,52}$ Other studies using animal models have shown that directly blocking $\beta 3$ integrin inhibits angiogenesis and tumor growth. ${ }^{14,15,53,54}$ In contrast, work examining ovarian carcinomas has shown that high levels of $\beta 3$ integrin correlated with improved clinical outcome and that reducing $\beta 3$ integrin expression enhanced ovarian tumor growth. ${ }^{55}$ These seemingly opposing functions for $\beta 3$ integrin might 
be explained by the fact that the final outcome of $\beta 3$ integrin signaling within a particular cell or tumor type depends, in part, on the nature of the different ligands binding to this integrin, and the types of adaptor molecules that are assembled into signaling complexes that facilitate transmission of extracellular cues. For example, it is well established that certain $\beta 3$ integrin binding ligands, such as vitronectin, osteopontin, and periostin, can induce protumorigenic signaling events. ${ }^{56-58}$ In contrast, other bioactive factors that bind to $\beta 3$ integrin, such as tumstatin, canstatin, and endostatin, have been shown to inhibit tumor growth. ${ }^{59-61}$ Interestingly, overexpression of $\beta 3$ integrin in some ovarian tumor cell lines resulted in reduced proliferation that was associated with a $\mathrm{G}_{2} / \mathrm{M}$ block in the cell cycle and altered phosphorylation of CD2. ${ }^{55}$ In other studies, evidence suggests that unoccupied integrin $\alpha \mathrm{v} \beta 3$ may result in signaling, leading to a process termed integrin-mediated cell death. ${ }^{62}$ The ability of $\alpha v \beta 3$ to control distinct functions in different cell types, such as immune cells, may also impact the overall contribution of $\alpha \mathrm{v} \beta 3$ to tumor growth. For example, $\alpha v \beta 3$ expressed in macrophages and dendritic cells has been shown to play roles in phagocytosis of tumor cells as well as promoting antigen presentation. ${ }^{63,64}$ In addition, $\alpha v \beta 3$ has also been shown to play a role in regulating T-cell receptor signaling and motility, ${ }^{65,66}$ all of which may depend on the molecular features of the different ligands that bind to this integrin. In fact, studies have shown that direct targeting of $\beta 3$ or reducing $\beta 3$ integrin in a subpopulation of immune cells results in the induction of immune suppression, ${ }^{63,64}$ thereby compromising the efficacy of this therapeutic strategy as it relates to tumor growth. Therefore, designing and evaluating alternative strategies to disrupt $\alpha \mathrm{v} \beta 3$-dependent signaling without directly targeting $\alpha v \beta 3$ itself may help in the development of more effective treatments.

Our findings and those from many other studies are consistent with the notion that the overall contribution of $\alpha v \beta 3$ to tumor growth depends on a complex and interconnected network of parameters that include the cell type that the integrin is functioning in, the molecular characteristics of the local $\alpha \mathrm{v} \beta 3$ binding ligands, and the bioavailability and composition of the downstream signaling molecules required for the formation of intracellular signaling complexes that facilitate activation of distinct signaling cascades. Given this complexity, a therapeutic strategy of directly targeting all $\alpha v \beta 3$ in different cell types may not represent an optimal approach. As an alternative strategy, we have been studying whether selective targeting of discrete protumorigenic ligands of integrin $\alpha v \beta 3$, instead of directly targeting the integrin itself, may provide a clinically effective new approach to control protumorigenic signaling events. This alternative strategy might allow for selective inhibition of protumorigenic stimuli mediated through integrin $\alpha \mathrm{v} \beta 3$ and, at the same time, allow for the engagement of locally available tumor suppressing ligands to simulate inhibitory signals through $\alpha \mathrm{v} \beta 3$ within distinct stromal and tumor cell populations, ultimately promoting a more robust therapeutic outcome when used either alone or in combination with other cancer treatments. Taken together, a therapeutic strategy that focuses on selectively targeting a novel protumorigenic bioactive ligand of $\alpha \mathrm{v} \beta 3$ integrin, rather than directly targeting the $\alpha \mathrm{v} \beta 3$ receptor itself, may help selectively disrupt protumorigenic signaling pathways operating within both tumor and stroma cells to allow for an effective treatment paradigm for managing ovarian cancer.

\section{Supplemental Data}

Supplemental material for this article can be found at http://doi.org/10.1016/j.ajpath.2020.11.009.

\section{References}

1. Ghoneum A, Afify H, Salih Z, Kelly M, Said N: Role of tumor microenvironment in the pathobiology of ovarian cancer: insights and therapeutic opportunities. Cancer Med 2018, 7:5047-5056

2. Baghban R, Roshangar L, Jahanban-Esfahlan R, Seidi K, EbrahimiKalan A, Jaymand M, Kolahian S, Javaheri T, Zare P: Tumor microenvironment complexity and therapeutic implications at a glance. Cell Commun Signal 2020, 18:59

3. Musrap N, Diamandis EP: Revisiting the complexity of the ovarian cancer microenvironment-clinical implications for treatment strategies. Mol Cancer Res 2012, 10:1254-1264

4. Hamidi H, Ivaska J: Every step of the way: integrins in cancer progression and metastasis. Nat Rev Cancer 2018, 18:533-548

5. Paolillo M, Schinelli S: Extracellular matrix alterations in metastatic processes. Int J Mol Sci 2019, 20:4947

6. Janiszewska M, Primi MC, Izard T: Cell adhesion in cancer: beyond the migration of single cells. J Biol Chem 2020, 295:2495-2505

7. Xu J, Rodriguez D, Petitclerc E, Kim JJ, Hangai M, Moon YS, Davis GE, Brooks PC: Proteolytic exposure of a cryptic site within collagen type IV is required for angiogenesis and tumor growth in vivo. J Cell Biol 2001, 154:1069-1079

8. Akalu A, Roth JM, Caunt M, Policarpio D, Liebes L, Brooks PC: Inhibition of angiogenesis and tumor metastasis by targeting a matrix immobilized cryptic extracellular matrix epitope in laminin. Cancer Res 2007, 67:4353-4363

9. Caron JM, Ames JJ, Contois L, Liebes L, Friesel R, Muggia F, Vary CP, Oxburgh L, Brooks PC: Inhibition of ovarian tumor growth by targeting the HU177 cryptic collagen epitope. Am J Pathol 2016, 186:1649-1661

10. Ricard-Blum S, Vallet SD: Fragments generated upon extracellular matrix remodeling: biological regulators and potential drugs. Matrix Biol 2019, 75-76:170-189

11. Ames JJ, Contois L, Caron JM, Tweedie E, Yang X, Friesel R, Vary C, Brooks PC: Identification of an endogenously generated cryptic collagen epitope (XL313) that may selectively regulate angiogenesis by an integrin yes-associated protein (YAP) mechanotransduction pathway. J Biol Chem 2016, 291:2731-2750

12. Kehlet SN, Sanz-Pamplona R, Brix S, Leeming DJ, Karsdal MA, Moreno V: Excessive collagen turnover products are released during colorectal cancer progression and elevated in serum from metastatic colorectal cancer patients. Sci Rep 2016, 6:30599

13. Jensen C, Madsen DH, Hansen M, Schmidt H, Svane IM, Karsdal MA, Willumsen N: Non-invasive biomarkers derived from the extracellular matrix associate with response to immune 
checkpoint blockade (anti-CTLA-4) in metastatic melanoma patients J Immunother Cancer 2018, 6:152

14. Brooks PC, Montgomery AM, Rosenfeld M, Reisfeld RA, Hu T, Klier G, Cheresh DA: Integrin alpha v beta 3 antagonists promote tumor regression by inducing apoptosis of angiogenic blood vessels. Cell 1994, 79:1157-1164

15. Brooks PC, Clark RA, Cheresh DA: Requirement of vascular integrin alpha v beta 3 for angiogenesis. Science 1994, 264:569-571

16. Zanconato F, Cordenonsi M, Piccolo S: YAP and TAZ: a signaling hub of the tumour microenvironment. Nat Rev Cancer 2019, 19: 454-464

17. Lin X, Spindler TJ, de Souza Fonseca MA, Corona RI, Seo JH, Dezem FS, Li L, Lee JM, Long HW, Sellers TA, Karlan BY, Noushmehr H, Freedman ML, Gayther SA, Lawrenson K: Super-enhancer-associated LncRNA UCA1 interacts directly with AMOT to activate YAP target genes in epithelial ovarian cancer. iScience 2019, 17:242-255

18. Yuan M, Tomlinson V, Lara R, Holliday D, Chelala C, Harada T, Gangeswaran R, Manson-Bishop C, Smith P, Danovi SA, Pardo O, Crook T, Mein CA, Lemoine NR, Jones LJ, Basu S: Yes-associated protein (YAP) functions as a tumor suppressor in breast. Cell Death Differ 2008, 15:1752-1759

19. Moya IM, Castaldo SA, Van den Mooter L, Soheily S, SansoresGarcia L, Jacobs J, Mannaerts I, Xie J, Verboven E, Hillen H, AlgueroNadal A, Karaman R, Van Haele M, Kowalczyk W, De Waegeneer M, Verhulst S, Karras P, van Huffel L, Zender L, Marine JC, Roskams T, Johnson R, Aerts S, van Grunsven LA, Halder G: Peritumoral activation of the hippo pathway effectors YAP and TAZ suppresses liver cancer in mice. Science 2019, 366:1029-1034

20. Misra JR, Irvine KD: The hippo signaling network and its biological functions. Annu Rev Genet 2018, 52:65-87

21. Mo JS: The role of extracellular biophysical cues in modulating the Hippo-YAP pathway. BMB Rep 2017, 50:71-78

22. Anderson KG, Voillet V, Bates BM, Chiu EY, Burnett MG, Garcia NM, Oda SK, Morse CB, Stromnes IM, Drescher CW, Gottardo R, Greenberg PD: Engineered adoptive T-cell therapy prolongs survival in a preclinical model of advanced-stage ovarian cancer. Cancer Immunol Res 2019, 7:1412-1425

23. Blystone SD: Kinetic regulation of beta 3 integrin tyrosine phosphorylation. J Biol Chem 2002, 277:46886-46890

24. Caron JM, Han X, Contois L, Vary CPH, Brooks PC: The HU177 collagen epitope controls melanoma cell migration and experimental metastasis by a CDK5/YAP-dependent mechanism. Am J Pathol 2018, 188:2356-2368

25. Cosset E, Ilmjarv S, Dutoit V, Elliott $\mathrm{K}$, von Schalscha $\mathrm{T}$, Camargo MF, Reiss A, Moroishi T, Seguin L, Gomez G, Moo JS, Preynat-Seauve O, Krause KH, Chneiweiss H, Sarkaria JN, Guan KL, Dietrich PY, Weis SM, Mischel PS, Cheresh DA: Glut3 addiction is a druggable vulnerability for a molecularly defined subpopulation of glioblastoma. Cancer Cell 2017, 32:856-868

26. Elbediwy A, Vincent-Mistiaen ZI, Spencer-Dene B, Stone RK, Boeing S, Wculek SK, Cordero J, Tan EH, Ridgway R, Brunton VG, Sahai E, Gerhardt H, Behrens A, Malanchi I, Sansom OJ, Thompson BJ: Integrin signaling regulates YAP and TAZ to control skin homeostasis. Development 2016, 143:1674-1687

27. Zhou Y, Huang T, Cheng AS, Yu J, Kang W, To KF: The TEAD family and its oncogenic role in promoting tumorigenesis. Int J Mol Sci 2016, 17:138

28. Zanconato F, Forcato M, Battilana G, Azzolin L, Quaranta E, Bodega B, Rosato A, Bicciato S, Cordenonsi M, Piccolo S: Genome-wide association between YAP/TAZ/TEAD and AP-1 at enhancers drives oncogenic growth. Nat Cell Biol 2015, 17: $1218-1227$

29. Tashiro H, Miyazaki K, Okamura H, Iwai A, Fukumoto M: C-MYC over-expression in human primary ovarian tumours: its relevance to tumour progression. Int J Cancer 1992, 50:828-833

30. Prathapam T, Aleshin A, Guan Y, Gray JW, Martin GS: p27Kip1 mediates addiction of ovarian cancer cells to MYCC (c-MYC) and their dependence on MYC paralogs. J Biol Chem 2010, 285: 32529-32538

31. Hao Y, Chun A, Cheung K, Rashidi B, Yang X: Tumor suppressor LATS1 is a negative regulator of oncogene YAP. J Biol Chem 2008, 283:5496-5509

32. St John MA, Tao W, Fei X, Fukumoto R, Carcangiu ML, Brownstein DG, Parlow AF, McGrath J, Xu T: Mice deficient of Lats1 develop soft-tissue sarcomas, ovarian tumours and pituitary dysfunction. Nat Genet 1999, 21:182-186

33. Si Y, Ji X, Cao X, Dai X, Xu L, Zhao H, Guo X, Yan H, Zhang H, Zhu C, Zhou Q, Tang M, Xia Z, Li L, Cong YS, Ye S, Liang T, Feng XH, Zhao B: Src inhibits the hippo tumor suppressor pathway through tyrosine phosphorylation of Lats1. Cancer Res 2017, 77: 4868-4880

34. Lamar JM, Xiao Y, Norton E, Jiang ZG, Gerhard GM, Kooner S, Warren JSA, Hynes RO: SRC tyrosine kinase activates the YAP/TAZ axis and thereby drives tumor growth and metastasis. J Biol Chem 2019, 294:2302-2317

35. Ege N, Dowbaj AM, Jiang M, Howell M, Hooper S, Foster C, Jenkins RP, Sahai E: Quantitative analysis reveals that actin and Src-family kinases regulate nuclear YAP1 and its export. Cell Syst 2018, 6:692-708

36. Natarajan S, Foreman KM, Soriano MI, Rossen NS, Shehade H, Fregoso DR, Eggold JT, Krishnan V, Dorigo O, Krieg AJ, Heilshorn SC, Sinha S, Fuh KC, Rankin EB: Collagen remodeling in the hypoxic tumor-mesothelial niche promotes ovarian cancer metastasis. Cancer Res 2019, 79:2271-2284

37. Lengyel E: Ovarian cancer development and metastasis. Am J Pathol 2010, 177:1053-1064

38. De Nola R, Menga A, Castegna A, Loizzi V, Ranieri G, Cicinelli E, Cormio G: The crowded crosstalk between cancer cells and stromal microenvironment in gynecological malignancies: biological pathways and therapeutic implication. Int J Mol Sci 2019, 20:2401

39. Reggiani F, Gobbi G, Ciarrocchi A, Ambrosetti DC, Sancisi V: Multiple roles and context-specific mechanisms underlying YAP and TAZ-mediated resistance to anti-cancer therapy. Biochim Biophys Acta Rev Cancer 2020, 1873:188341

40. Low BC, Pan CQ, Shivashankar GV, Bershadsky A, Sudol M, Sheetz M: YAP/TAZ as mechanosensors and mechanotransducers in regulating organ size and tumor growth. FEBS Lett 2014, 588:2663-2670

41. Dasgupta I, McCollum D: Control of cellular responses to mechanical cues through YAP/TAZ regulation. J Biol Chem 2019, 294: 17693-17706

42. Heallen T, Zhang M, Wang J, Bonilla-Claudio M, Klysik E, Johnson RL, Martin JF: Hippo pathway inhibits Wnt signaling to restrain cardiomyocyte proliferation and heart size. Science 2011, 332:458-461

43. Zhou D, Conrad C, Xia F, Park JS, Payer B, Yin Y, Lauwers GY, Thasler W, Lee JT, Avruch J, Bardeesy N: Mst1 and Mst2 maintain hepatocyte quiescence and suppress hepatocellular carcinoma development through inactivation of the Yap1 oncogene. Cancer Cell 2009, 16:425-438

44. Patel SH, Camargo FD, Yimlamai D: Hippo signaling in the liver regulates organ size, cell fate, and carcinogenesis. Gastroenterology 2017, 152:533-545

45. Zhou D, Zhang Y, Wu H, Barry E, Yin Y, Lawrence E, Dawson D, Willis JE, Markowitz SD, Camargo FD, Avruch J: Mst1 and Mst2 protein kinases restrain intestinal stem cell proliferation and colonic tumorigenesis by inhibition of Yes-associated protein (Yap) overabundance. Proc Natl Acad Sci U S A 2011, 108:E1312-E1320

46. Yu FX, Meng Z, Plouffe SW, Guan KL: Hippo pathway regulation of gastrointestinal tissues. Annu Rev Physiol 2015, 77:201-227

47. George NM, Day CE, Boerner BP, Johnson RL, Sarvetnick NE: Hippo signaling regulates pancreas development through inactivation of Yap. Mol Cell Biol 2012, 32:5116-5128

48. Feng X, Degese MS, Iglesias-Bartolome R, Vaque JP, Molinolo AA, Rodrigues M, Zaidi MR, Ksander BR, Merlino G, Sodhi A, Chen Q, 
Gutkind JS: Hippo-independent activation of YAP by the GNAQ uveal melanoma oncogene through a trio-regulated rho GTPase signaling circuitry. Cancer Cell 2014, 25:831-845

49. Shao DD, Xue W, Krall EB, Bhutkar A, Piccioni F, Wang X, Schinzel AC, Sood S, Rosenbluh J, Kim JW, Zwang Y, Roberts TM, Root DE, Jacks T, Hahn WC: KRAS and YAP1 converge to regulate EMT and tumor survival. Cell 2014, 158:171-184

50. Barbolina MV, Adley BP, Ariztia EV, Liu Y, Stack MS: Microenvironmental regulation of membrane type 1 matrix metalloproteinase activity in ovarian carcinoma cells via collagen-induced EGR1 expression. J Biol Chem 2007, 282:4924-4931

51. Kageshita T, Hamby CV, Hirai S, Kimura T, Ono T, Ferrone S: Alpha(v)beta3 expression on blood vessels and melanoma cells in primary lesions: differential association with tumor progression and clinical prognosis. Cancer Immunol Immunother 2000, 49:314-318

52. Gasparini G, Brooks PC, Biganzoli E, Vermeulen PB, Bonoldi E, Dirix LY, Ranieri G, Miceli R, Cheresh DA: Vascular integrin alpha(v)beta3: a new prognostic indicator in breast cancer. Clin Cancer Res 1998, 4:2625-2634

53. Petitclerc E, Stromblad S, von Schalscha TL, Mitjans F, Piulats J, Montgomery AM, Cheresh DA, Brooks PC: Integrin alpha(v)beta3 promotes M21 melanoma growth in human skin by regulating tumor cell survival. Cancer Res 1999, 59:2724-2730

54. Brooks PC, Stromblad S, Klemke R, Visscher D, Sarkar FH, Cheresh DA: Antiintegrin alpha v beta 3 blocks human breast cancer growth and angiogenesis in human skin. J Clin Invest 1995, 96: $1815-1822$

55. Kaur S, Kenny HA, Jagadeeswaran S, Zillhardt MR, Montag AG, Kistner E, Yamada SD, Mitra AK, Lengyel E: \{beta\}3-Integrin expression on tumor cells inhibits tumor progression, reduces metastasis, and is associated with a favorable prognosis in patients with ovarian cancer. Am J Pathol 2009, 175:2184-2196

56. Burgos-Panadero R, Noguera I, Canete A, Navarro S, Noguera R: Vitronectin as a molecular player of the tumor microenvironment in neuroblastoma. BMC Cancer 2019, 19:479

57. Sharon Y, Raz Y, Cohen N, Ben-Shmuel A, Schwartz H, Geiger T, Erez N: Tumor-derived osteopontin reprograms normal mammary fibroblasts to promote inflammation and tumor growth in breast cancer. Cancer Res 2015, 75:963-973

58. Chen G, Nakamura I, Dhanasekaran R, Iguchi E, Tolosa EJ, Romecin PA, Vera RE, Almada LL, Miamen AG, Chaiteerakij R, Zhou M, Asiedu MK, Moser CD, Han S, Hu C, Banini BA,
Oseini AM, Chen Y, Fang Y, Yang D, Shaleh HM, Wang S, Wu D, Song T, Lee JS, Thorgeirsson SS, Chevet E, Shah VH, FernandezZapico ME, Roberts LR: Transcriptional induction of periostin by a sulfatase 2-TGFbeta1-SMAD signaling axis mediates tumor angiogenesis in hepatocellular carcinoma. Cancer Res 2017, 77: 632-645

59. Hamano Y, Zeisberg M, Sugimoto H, Lively JC, Maeshima Y, Yang C, Hynes RO, Werb Z, Sudhakar A, Kalluri R: Physiological levels of tumstatin, a fragment of collagen IV alpha3 chain, are generated by MMP-9 proteolysis and suppress angiogenesis via alphaV beta3 integrin. Cancer Cell 2003, 3:589-601

60. Kamphaus GD, Colorado PC, Panka DJ, Hopfer H, Ramchandran R, Torre A, Maeshima Y, Mier JW, Sukhatme VP, Kalluri R: Canstatin, a novel matrix-derived inhibitor of angiogenesis and tumor growth. J Biol Chem 2000, 275:1209-1215

61. Rehn M, Veikkola T, Kukk-Valdre E, Nakamura H, Ilmonen M, Lombardo C, Pihlajaniemi T, Alitalo K, Vuori K: Interaction of endostatin with integrins implicated in angiogenesis. Proc Natl Acad Sci U S A 2001, 98:1024-1029

62. Stupack DG, Puente XS, Boutsaboualoy S, Storgard CM, Cheresh DA: Apoptosis of adherent cells by recruitment of caspase- 8 to unligated integrins. J Cell Biol 2001, 155:459-470

63. Su X, Esser AK, Amend SR, Xiang J, Xu Y, Ross MH, Fox GC, Kobayashi T, Steri V, Roomp K, Fontana F, Hurchla MA, Knolhoff BL, Meyer MA, Morgan EA, Tomasson JC, Novack JS, Zou W, Faccio R, Novack DV, Robinson SD, Teitelbaum SL, DeNardo DG, Schneider JG, Weilbaecher KN: Antagonizing integrin beta3 increases immunosuppression in cancer. Cancer Res 2016, 76:3484-3495

64. Jinushi M, Chiba S, Baghdadi M, Kinoshita I, Dosaka-Akita H, Ito $\mathrm{K}$, Yoshiyama $\mathrm{H}$, Yagita $\mathrm{H}$, Uede $\mathrm{T}$, Takaoka A: ATMmediated DNA damage signals mediate immune escape through integrin-alphavbeta3-dependent mechanisms. Cancer Res 2012, 72:56-65

65. Doucey MA, Legler DF, Faroudi M, Boucheron N, Baumgaertner P, Naeher D, Cebecauer M, Hudrisier D, Ruegg C, Palmer E, Valitutti S, Bron C, Luescher IF: The beta1 and beta3 integrins promote $\mathrm{T}$ cell receptor-mediated cytotoxic T lymphocyte activation. J Biol Chem 2003, 278:26983-26991

66. Neto EH, Coelho AL, Sampaio AL, Henriques M, Marcinkiewicz C, De Freitas MS, Barja-Fidalgo C: Activation of human T lymphocytes via integrin signaling induced by RGD-disintegrins. Biochim Biophys Acta 2007, 1773:176-184 